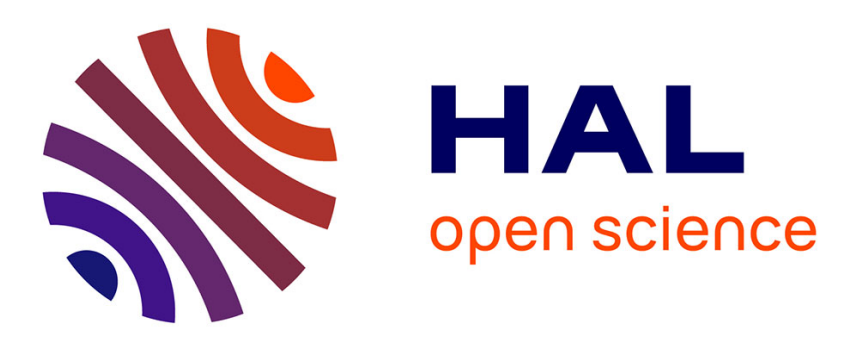

\title{
An adaptive finite element method for the modeling of the equilibrium of red blood cells
}

Aymen Laadhari, Pierre Saramito, Chaouqi Misbah

\section{To cite this version:}

Aymen Laadhari, Pierre Saramito, Chaouqi Misbah. An adaptive finite element method for the modeling of the equilibrium of red blood cells. International Journal for Numerical Methods in Fluids, 2016, 80 (7), pp.397-428. 10.1002/fld.4086 . hal-01071440

\author{
HAL Id: hal-01071440 \\ https://hal.science/hal-01071440
}

Submitted on 14 Oct 2014

HAL is a multi-disciplinary open access archive for the deposit and dissemination of scientific research documents, whether they are published or not. The documents may come from teaching and research institutions in France or abroad, or from public or private research centers.
L'archive ouverte pluridisciplinaire $\mathbf{H A L}$, est destinée au dépôt et à la diffusion de documents scientifiques de niveau recherche, publiés ou non, émanant des établissements d'enseignement et de recherche français ou étrangers, des laboratoires publics ou privés.

\section{()ㅜ) $\Theta$}

Distributed under a Creative Commons Attribution - NoDerivatives| 4.0 International 


\title{
An Adaptive Finite Element Method For The Modeling Of The Equilibrium Of Red Blood Cells
}

\author{
Aymen Laadhari ${ }^{\mathrm{a}, *}$, Pierre Saramito ${ }^{\mathrm{b}}$, Chaouqi Misbah $^{\mathrm{c}}$ \\ ${ }^{a}$ Computer Vision Laboratory, Institut für Bildverarbeitung, Department of Information Technology and Electrical Engineering, Swiss \\ Federal Institute of Technology - ETHZ, CH-8092 Zürich, Switzerland. \\ ${ }^{b}$ Laboratoire Jean Kuntzmann, Informatique et Mathématiques Appliquées de Grenoble, Université Joseph Fourier and Centre \\ National de la Recherche Scientifique, F-38041 Grenoble, France. \\ ${ }^{c}$ Laboratoire Interdisciplinaire de Physique, Université Joseph Fourier and Centre National de la Recherche Scientifique, F-38041 \\ Grenoble, France.
}

\begin{abstract}
This contribution is concerned with a the numerical modeling of an isolated red blood cell (RBC), and more generally of phospholipid membranes. We propose an adaptive Eulerian finite element approximation, based on the level set method, of a shape optimization problem arising in the study of RBC's equilibrium. We simulate the equilibrium shapes that minimize the elastic bending energy under prescribed constraints of fixed volume and surface area. An anisotropic mesh adaptation technique is used in the vicinity of the cell's membrane to enhance the robustness of the method. Efficient time and spatial discretizations are considered and implemented. We address in detail the main features of the proposed method and finally we report several numerical experiments in the two-dimensional and the three-dimensional axisymmetric cases. The effectiveness of the numerical method is further demonstrated through numerical comparisons with semi-analytical solutions provided by a reduced order model.
\end{abstract}

Keywords: Level set method, Finite element approximation, Mesh adaptation, Minimization under constraints, Lagrange multipliers, Numerical methods, Vesicle, Red Blood Cell, Elastic bending energy, Canham and Helfrich model

\section{Introduction}

The blood represents an essential element for life. It is composed of several cell types which possess specific functions. In particular, red blood cells (RBCs) are the most abundant cells in the blood and allow to carry oxygen through the body. Blood disorders, such as sickle-cell disease, hemophilia, anemia, polycythemia vera and hemophilia, represent an important problem in public health that affect millions of people each year and resulting in several complications. Advancing the modeling strategies and the computational methodologies of the blood function can certainly be a key component in the understanding of blood disorders and the development of novel therapies and prognostic methods. Since several decades, there is an increasing interest in many aspects of blood modeling driven by the increasing demand from the medical community for scientifically rigorous studies of blood. Scientifical investigations extend to a wide range of spatio-temporal scales covering the microscopic and the macroscopic scales. In the present work, we are interested in the microscopic modeling of RBCs. Vesicles, also called liposomes, represent a simple and attractive model introduced to mimic the viscoelastic and the mechanical behaviors of RBCs. They are closed membranes having the structure of amphiphilic molecules that are self-assembled in water to build a structure of bilayers. Regarding the modeling of RBCs and vesicles, several investigations and substantial achievements have been made involving researchers from diverse communities in the fields of biology [61, 70], applied mathematics [7, 45, 65, 44, 8], scientific computing [25, 68, 23] and the biomedical field [60]. These cells exhibit a wide and rich set of shapes in various physical environments. Effective mathematical models,

\footnotetext{
* Corresponding author

Email addresses: laadhari@vision.ee.ethz.ch (Aymen Laadhari), Pierre.Saramito@imag.fr (Pierre Saramito), cmisbah@ujf-grenoble.fr (Chaouqi Misbah)
} 
seconded by the use of accurate numerical methodologies, are needed to study, in particular, the equilibrium shapes of RBCs.

A wide variety of models was developed to describe the deformation of cell membranes in the steady flow and under the effect of the surrounding fluid dynamics, see e.g. [16, 17, 15, 43, 79, 44, 52, 65, 39, 44, 69]. Because of the incompressibility, the main mode of the deformation of vesicles is bending, and the bending energy (or elastic energy) describes the cell shapes. In the early 1970s, Canham [12] and Helfrich [72, 57] formalized the physical properties of the cell membrane in a mathematical model. They introduced a model in which the cost in the bending energy is given by the curvature of the membrane. Let us define the mean curvature $H$ and the Gauss curvature $K$, respectively, as the sum and the product of the principle curvatures defined on the cell membrane. Let $\Omega \in \mathbb{R}^{3}$ be a bounded domain with smooth boundary $\Gamma$ that represents the cell membrane. Canham and Helfrich introduced the following expression of the bending energy, called also Willmore energy [11, 73]

$$
\mathcal{J}=\frac{k}{2} \int_{\Gamma}\left(H-H_{0}\right)^{2} \mathrm{~d} s+\frac{k_{g}}{2} \int_{\Gamma} K \mathrm{~d} s,
$$

where the parameters $k$ and $k_{g}$ are two constants that represent the bending rigidity and the Gaussian curvature modulus, respectively. In the present work, we assume unit values of $k$ and $k_{g}$. The spontaneous curvature $H_{0}$ allows to describe the asymmetry effect of the membrane and its surrounding environment, which is caused by inhomogeneities within the structure of the membrane bilayers, see e.g. [71]. The spontaneous curvature has no effect on the shape of the cell in the two-dimensional case, whereas the shape shall depend on $H_{0}$ in the tridimensional case, see [49]. In the expression of the bending energy, the Gauss curvature $K$ component allows to consider the topological changes of the cell. Using the Gauss-Bonnet theorem, the second integral of the bending energy $\mathcal{J}$ is a topological invariant. We make the assumption that the shape topological gendre is preserved because topological changes are energetically disadvantaged, see e.g. [21, 19, 49]. Since no topological changes are allowed, the integral component of $\mathcal{J}$ considering the Gauss curvature is disregarded.

The membranes of vesicles, or red blood cells, have special properties that drive the cell dynamics and control the equilibrium forms. Indeed, the cell's membrane is considered impermeable and the number of molecules in each layer remains fixed. Accordingly, the cost of the bending deformations remains drastically smaller than the cost of stretching or compressing the membrane. Therefore, the properties of incompressibility of the inner domain of the cell, and the inextensibility of the membrane are assumed. The equilibrium configurations of RBCs, and vesicles, shall represent the minimizers of the energy of Canham and Helfrich constrained to have fixed inner volume and fixed surface area. As a consequence, a shape optimization problem exists, and the shape of the RBC depends on specific parameters that are obtained by the non-dimensional formulation of the problem. The RBCs in their natural environment can be more or less inflated, and the deflation could be due to the thermal expansion of phospholipids or the osmotic effects. Let us introduce the area $A_{0}$ and the volume $V_{0}$ of the cell. A shape parameter, called the reduced volume and denoted by $\gamma$, is a dimensionless parameter that measures the deflation of the cell. It is defined as the ratio between the volume of the cell and the volume of a sphere having the surface area $A_{0}$.

$$
\gamma=\frac{3 V_{0}}{4 \pi} \times\left(\frac{4 \pi}{A_{0}}\right)^{3 / 2}
$$

Accordingly, the reduced volume can range from 0, i.e. completely deflated shape, to 1, i.e. spherecal shape. Human RBCs have usually a biconcave disk-like shape having a reduced volume $\gamma \approx 0.64$. However, several other shapes are observed by varying the shape parameter and we have, for instance, the stomatocytes, the oblates and the prolates, see e.g [66, [6]. In the two-dimensional case, the dimensionless shape parameter is called the reduced area and it is given by the ratio between the cell area and the area of a circle having the same perimeter as the cell. The reduced area can range also from 0 to 1, i.e. circular shape, and it is expressed in the two-dimensional case by

$$
\gamma=\frac{V_{0}}{\pi} \times\left(\frac{2 \pi}{A_{0}}\right)^{2}
$$

The minimization of the bending energy $\mathcal{J}$ under the constraints of fixed volume and area yields the equilibrium shapes. The equilibrium condition is called commonly the Euler-Lagrange equation, and it uses two Lagrange multipliers $p$ and $\sigma$ to impose the volume and the area constraints, respectively. This relation was obtained using various mathematical approaches, such as the differential geometry, see e.g. [80, 18], and the shape differentiation technique, see e.g. 67, 1, 47]. The mechanical equilibrium equation of the cell membrane reads

$$
p+\sigma H+k_{c}\left(\frac{1}{2}\left(H-H_{0}\right)\left[4 K-H\left(H+H_{0}\right)\right]-\Delta_{s} H\right)=0 .
$$


The Laplace-Beltrami operator is denoted by $\Delta_{s}$, see Section 2, Several experimental and theoretical studies have focused on the investigation of the configurations of equilibrium of BRCs and vesicles . While there is a substantial interest from the point of view of scientific computing and numerical methods, most of these contributions are based on the study of lumped models, ie constituted by ordinary differential equations. Some numerical strategies for the modeling of RBCs shapes in the equilibrium can be found in [65, 61, 70, 21, 22]. The membrane represents a free interface, and there are basically two categories of numerical methods to follow the evolution of the membrane to the position of static equilibrium. The problem can be formulated in a total Lagrangian setting, where equations have to be written in the reference configuration. In such case, the cell membrane is fitted by a Lagrangian mesh, and it deforms according to its motion that minimizes the bending energy. Although more precise, the Lagrangian methods are often a demanding task that could generate computational instabilities related to the deterioration in the quality of the mesh. Hence, sophisticated remeshing strategies should be used in most usual cases. For instance, we can cite the boundary element method, see e.g. [14, 46], and the immersed boundary method developed by Peskin, see e.g. [59]. In an alternative framework, computational difficulties associated with e.g. the mesh distortion can be avoided using fully Eulerian methods, where the interface is then implicitly captured and it never has to be explicitly reconstructed. Within this framework, a good solver for the advection problem is required, and the advection of the cell membrane shall be done with high accuracy. Additional numerical difficulties related to an excessive amount of mass loss or gain must be handled [77]. A single set of governing equations must be solved in the entire computational domain using usually a fixed mesh. In the existing bibliography, various methods have been used to model free interfaces motion in an Eulerian framework. For instance, we cite the volume-of-fluid method, see e.g. [37, 51], the phase field method, see e.g. [2, 20], and the level set method, see e.g. 56, 55]). In the present work, we use the level set method, as in [56, 13], where the cell membrane is implicitly described as a particular level set. The level set function has the specificity to have a nearly unit gradient. Our development has been further motivated by [22], where an energetic variationnal formulation was introduced using an interface diffuse model.

Regarding the membrane incompressibility, it plays an essential role to get the observed shapes of RBCs. It helps to preserve a fixed surface area that represents a global constraint for the shape optimization problem. This constraint could be satisfied using a penalty approach, where constraint is not exactly imposed, and the computed solution depends on the penalty parameter, see e.g. [13, 21]. The second strategy introduces a global Lagrange multiplier that ensures exactly the inextensibility constraint, see e.g. [22]. The second strategy is considered in this work. In what follows, we describe a numerical model to simulate the evolution of the cell membrane to the equilibrium. The equilibrium state corresponds to the minimization of the bending energy under prescribed values of volume and surface area. An Eulerian finite element method based on the level set approach is used. To overcome a usual drawback of Eulerian methods, we first employ a modified level set approach based on the imposition of additional constraints via Lagrange multipliers that helps to enforce the conservation of mass. Furthermore, a mesh adaptation technique is used to better capture the cell membrane. A reduced order model helps to validate afterwards the computational results.

Outline. The paper is organized as follows. In Section 2, we introduce the required notations and we provide a consistent mathematical setting for the formulation of the physical model of minimal energy. A saddle-point formulation allows us to characterize the solution in the weak formulation. We present a detailed description of the mixed finite element method, and we describe an anisotropic mesh adaptation procedure that helps to enhance the mass preservation. We outline the detailed numerical algorithm in Section 3, and we provide the discretization schemes in time and space. We present in Section 4 a reduced order model that helps to validate our results. A set of numerical examples illustrating the main features of the model and the accuracy of the adaptive finite element computations are listed in Section 5. Finally, we make some concluding remarks in Section 6. Details on the reduced model in the axisymmetric case are reported in Appendix A.

\section{Mathematical model for RBC's equilibrium shape}

\subsection{Notations and mathematical setting}

Let $\Lambda \subset \mathbb{R}^{d}, d \in\{2,3\}$ be the bounded domain containing the RBC and the surrounding domain, where $L>0$ represents the domain width. Fig 1 provides a sketch of the cell domain and its position with respect to the computational domain. Let $\boldsymbol{x}$ represent the spatial coordinate in the domain $\Lambda$ at the time $t$. Let us introduce the outward unit normal vector $\mathbf{n}$ of $\Gamma$, see Fig. 1. Let us consider a scalar function $\varkappa$ and a vector field $\boldsymbol{u}$. We define the 


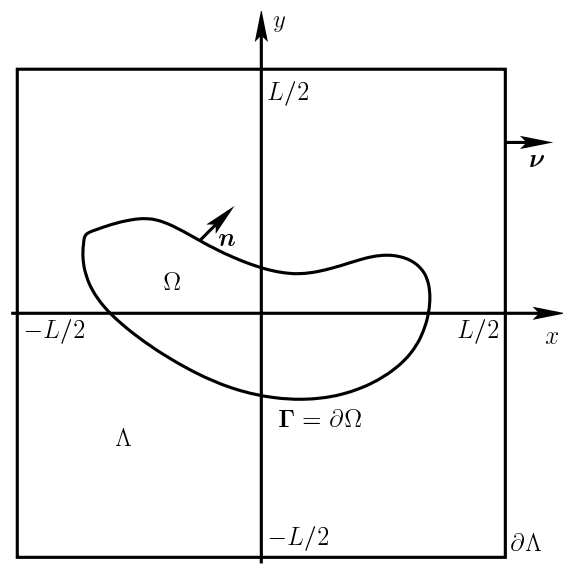

Figure 1: A sketch for the RBC embedded in the surrounding computational domain.

tangential gradient, the tangential divergence and the Laplace-Beltrami operator (called also surface Laplacian), that are given respectively by:

$$
\begin{aligned}
& \nabla_{s} \varkappa:=(\mathbf{I}-\mathbf{n} \otimes \mathbf{n}) \nabla \varkappa=\nabla \varkappa-(\mathbf{n} . \nabla \varkappa) \mathbf{n} \\
& \operatorname{div}_{s}(\mathbf{u}):=\operatorname{trace}\left(\nabla_{s} \varkappa\right)=(\mathbf{I}-\mathbf{n} \otimes \mathbf{n}): \nabla \mathbf{u} \\
& \Delta_{s} \varkappa:=\operatorname{div}_{s}\left(\nabla_{s} \varkappa\right)
\end{aligned}
$$

where the symbol $\otimes$ denotes the tensorial product, and the semicolon : represents the two times contracted product between tensors.

\subsection{Level set formulation}

We first notice that the time represents in the present article a numerical parameter introduced to describe the evolution of the shape to the equilibrium configuration, and it doesn't represent the physical time. Let $T>0$ and for any $t \in(0, T)$ let us assume that the domain within the cell has a smooth boundary $\Gamma=\partial \Omega$. The entire computational domain $\Lambda$ and the RBC's membrane are represented in Fig. 1. We introduce a level set function $\varphi$ defined in the domain $\Lambda$, in order to label the inside and the outside of the cell. The closed surface $\Gamma$ representing the membrane is defined by

$$
\Gamma=\{(t, \boldsymbol{x}) \in(0, T) \times \Lambda: \varphi(t, \boldsymbol{x})=0\} .
$$

Therefore, the membrane is a particular level set of $\varphi$ and, by convention, the set $\{(t, \boldsymbol{x}) \in(0, T) \times \Lambda: \varphi(t, \boldsymbol{x})<0\}$ represents the inner domain. Since the level set function is defined in the Eulerian configuration, the membrane motion is describes through the evolution of the level set function. The interface $\Gamma$ is transported with the same velocity, and thus the level set function satisfies

$$
\partial_{t} \varphi+\boldsymbol{u} \cdot \nabla \varphi=0 \text { in }(0, T) \times \Lambda .
$$

This equation is equipped with initial data and suitable boundary condition

$$
\varphi(0, \cdot)=\varphi_{0}(\cdot) \quad \text { in } \Lambda \quad \text { and } \quad \varphi=\varphi_{b} \quad \text { on }(0, T) \times \Sigma_{-},
$$

where the set $\Sigma_{-}=\{\boldsymbol{x} \in \partial \Lambda: \boldsymbol{u} \cdot \boldsymbol{\nu}(\boldsymbol{x})<0\}$ is the upstream boundary and $\boldsymbol{\nu}$ denotes the outward unit normal on the external boundary $\partial \Lambda$. The function $\varphi_{0}$ acts as initial datum for the transport equation, and it denotes the signed distance between $\boldsymbol{x} \in \Lambda$ and the initial position of the cell $\Gamma_{0}:=\partial \Omega_{0}$ :

$$
\varphi_{0}(\boldsymbol{x})= \begin{cases}\inf _{\boldsymbol{y} \in \Gamma_{0}}|\boldsymbol{y}-\boldsymbol{x}|, & \text { if } \boldsymbol{x} \notin \Omega_{0}, \\ -\inf _{\boldsymbol{y} \in \Gamma_{0}}|\boldsymbol{y}-\boldsymbol{x}|, & \text { otherwise. }\end{cases}
$$

The advection field $\boldsymbol{u}$ will be obtained afterwards from a shape optimization problem, and it will be encoded as a function of the level set $\varphi$. This will be the scope of the section 2.4 


\subsection{Redistancing and regularization}

By solving the advection problem (2.3), the level set function $\varphi$ loses its property of being a signed distance function, and $|\nabla \varphi| \neq 1 \in \Lambda$. We need to avoid this situation since too large or too small gradients of the level set function, notably around $\Gamma$ and $\partial \Lambda$, indicate respectively either a steep or a flat function whose zero is less accurately tracked. An auxiliary problem called commonly the redistancing problem may be solved to recover the signed distance property. An accurate redistancing procedure should keep some properties mainly: the inner volume $\Omega$ of the cell described by $\varphi<0$ should be preserved during the redistancing and the zero-level set, i.e. $\Gamma$, should keep its initial position. Although this problem was first introduced in [56], it is still not obvious to obtain satisfactory results. To address this difficulty, we introduce a modified redistancing problem where a Lagrange multiplier located in the vicinity of the interface is introduced to enhance the local mass conservation and to improve the performance of the method, see [48].

Let $\tilde{\varphi}(t,$.$) be the solution of (2.3) at time t \in(0, T)$ and we introduce a pseudo-time variable $\tau$. Initialized with $\tilde{\varphi}$, the redistancing problem consists in the resolution of the following Hamilton-Jacobi equation

$$
\begin{aligned}
\frac{\partial \varphi}{\partial \tau}(\tau, \boldsymbol{x} ; t)+\boldsymbol{v} \cdot \nabla \varphi(\tau, \boldsymbol{x} ; t) & =\operatorname{sgn}(\tilde{\varphi}(t, \boldsymbol{x}))+\lambda(\tau, x ; t) g(\varphi(\tau, \boldsymbol{x} ; t)) & & \text { a.e. in }(0,+\infty) \times \Lambda \\
\varphi(0, \boldsymbol{x} ; t) & =\tilde{\varphi}(t, \boldsymbol{x}) & & \text { a.e. in } \Lambda .
\end{aligned}
$$

The advection vector field writes $\boldsymbol{v}=\operatorname{sgn}(\tilde{\varphi}) \frac{\nabla \varphi}{|\nabla \varphi|}$, and $\operatorname{sgn}(\tilde{\varphi})$ denotes the sign function assuming the values $0,-1,+1$ on $\Gamma$, inside $\Omega$ and outside $\Omega$, respectively. In the computational level, a pseudo-stationary solution that approximates a signed distance function in the neighborhood of the membrane may be satisfactory. The Lagrange multiplier $\lambda(\tau, \boldsymbol{x} ; t)$ in introduced to enforce the constraint of a local constant volume at each $\boldsymbol{x} \in \Lambda$. The function $g(\varphi)$ localizes the mass correction in the vicinity of the interface, and it depends on the Dirac measure $\delta(\varphi)$. The Lagrange multiplier has an explicit average value $\lambda_{\mathcal{V}}$ over an arbitrary finite volume $\mathcal{V} \subset \Lambda$, see Fig. 4 (left):

$$
\lambda_{\mathcal{V}}(\tau ; t)=\frac{\int_{\mathcal{V}} \delta(\varphi)(\boldsymbol{v} \cdot \nabla \varphi-\operatorname{sgn}(\tilde{\varphi})) \varphi x}{\int_{\mathcal{V}} \delta(\varphi) g(\varphi)} \text { when } \mathcal{V} \cap \partial \Omega \neq \emptyset, \quad \text { and } \quad 0 \text { otherwise. }
$$

As a consequence, the stationary solution satisfies $|\nabla \varphi|=1$ almost everywhere in $\Lambda$, meaning that $\varphi(\infty, . ; t)$ is a signed distance and therefore it is taken as the new level set function $\varphi(t,$.$) . Therefore, the solution of (2.4)$ preserves the initial position $\Gamma$ for all pseudo-time $\tau>0$, and therefore the volume $|\Omega|$ is preserved as well.

For a given $\varepsilon>0$, a banded region of width $2 \varepsilon$ around $\Gamma$ is required. The regularization parameter $\varepsilon$ is chosen proportional to the mesh-size. The Heaviside function, the signum function and the Dirac measure shall respectively be substituted by smooth functions $\mathscr{H}_{\varepsilon}, \delta_{\varepsilon}$ and $\operatorname{sgn}_{\varepsilon}$. For all $\varphi \in \mathbb{R}$, they are, respectively, defined by

$$
\begin{aligned}
& \mathscr{H}_{\varepsilon}(\varphi)= \begin{cases}0, & \text { if } \varphi<-\varepsilon \\
\frac{1}{2}\left(1+\frac{\varphi}{\varepsilon}+\frac{\sin \left(\frac{\pi \varphi}{\varepsilon}\right)}{\pi}\right), & \text { if }|\varphi| \leq \varepsilon \quad \operatorname{sgn}_{\varepsilon}(\varphi)=2 \mathscr{H}_{\varepsilon}(\varphi)-1, \\
1, & \text { otherwise }\end{cases} \\
& \delta_{\varepsilon}(\varphi)=\frac{\mathrm{d} \mathscr{H}_{\varepsilon}}{\mathrm{d} \varphi}(\varphi)=\frac{1}{2 \varepsilon}\left(1+\cos \left(\frac{\pi \varphi}{\varepsilon}\right)\right) \quad \text { if } \quad|\varphi| \leq \varepsilon, \quad \text { and } \quad 0 \text { otherwise. }
\end{aligned}
$$

\subsection{Governing equations}

In this section we state the equations governing the model. We assume that the external domain of the cell is large enough so that the cell membrane does not touch the boundary $\partial \Lambda$. Let us consider the bending energy of Canham and Helfrich given at time $t \in(0, T)$ by:

$$
\mathcal{J}(\Omega)=\int_{\partial \Omega} \frac{k}{2}\left(H(\Omega)-H_{0}\right)^{2} \mathrm{~d} s .
$$

The RBC's membrane $\Gamma$ is described within the level set formulation, see Eq. 2.2. and the bending energy has to be encoded in terms of $\varphi$. Let us consider the space of the admissible solutions called $\mathbb{V}(\Omega)$. The equilibrium positions 
of RBCs are given by the shape optimization problem

$$
\Omega=\underset{w \in \mathbb{V}}{\operatorname{arginf}} \mathcal{J}(w) \quad \text { with } \mathbb{V}=\left\{\omega \subset \Lambda: \int_{\partial \omega} \mathrm{d} s=A_{0} \text { and } \int_{\omega} \mathrm{d} x=V_{0}\right\}
$$

where $A_{0}$ and $V_{0}$ represent the initial surface and the initial volume enclosed inside the cell, respectively at time $t=0$. In the two-dimensional case, these parameters represent the perimeter and the inner area of the cell, respectively. Moreover, we notice that the spontaneous curvature has no effect on the equilibrium shape in the two-dimensional case, see e.g. 21, 62, 49]. In what follows, we make the assumption that the spontaneous curvature $H_{0}$ is equal to zero. Furthermore, the minimization of the bending energy under the constraints of fixed volume and surface (2.7) is transformed into a saddle point formulation. The Lagrangian functional is introduced to relax the constraints.

$$
\mathcal{L}(\omega ; \sigma, q)=\mathcal{J}(\omega)+\sigma\left(\int_{\partial \omega} \mathrm{d} s-A_{0}\right)+p\left(\int_{\omega} \mathrm{d} x-V_{0}\right), \quad \forall(\omega ; \sigma, q),
$$

where $\sigma$ and $q$ represent the Lagrange multipliers corresponding respectively to the surface and the volume constraints. The solution $(\Omega ; p, \lambda) \in(\Lambda ; \mathbb{R}, \mathbb{R})$ is a saddle point of $\mathcal{L}$, and the problem writes

$$
(\Omega ; \lambda, p)=\underset{\omega \subset \Lambda}{\operatorname{arginf}} \sup _{\sigma \in \mathbb{R}} \mathcal{L}(\omega ; \sigma, q) .
$$

We consider the directional derivative of the Lagrangian functional along any directions $\mathbf{u}, q$ and $\mu$. The optimality condition is evaluated at $(\Omega ; p, \lambda)$ and it is given by

$$
\frac{\partial \mathcal{L}}{\partial \Omega}(\Omega ; \lambda, p)(\mathbf{u})=0 \quad \frac{\partial \mathcal{L}}{\partial p}(\Omega ; \lambda, p)(q)=0 \quad \frac{\partial \mathcal{L}}{\partial \lambda}(\Omega ; \lambda, p)(\mu)=0 .
$$

The deformation of the RBC is described by the advection in the direction of the steepest descent. In a previous work gradient descent vector, we derived a generalised mechanical equation describing the equilibrium of RBCs. Assuming a constant bending rigidity $k=1$, the Euler-Lagrange equation writes

$$
p+\lambda H+\frac{1}{2}\left(H-H_{0}\right)\left[4 K-H\left(H+H_{0}\right)\right]-\Delta_{s} H=0 .
$$

Using shape differentiation tools [53, 67, 1], the Fréchet derivative of (2.7) is computed in an arbitrary direction $\mathbf{u}$, see e.g. [20, 47], and it reads

$$
\frac{\partial \mathcal{L}}{\partial \omega}(\omega ; \lambda, p)(\mathbf{u})=\int_{\Gamma}\left\{p+\lambda H+\frac{1}{2}\left(H-H_{0}\right)\left[4 K-H\left(H+H_{0}\right)\right]-\Delta_{s} H\right\} \mathbf{u . n} \mathrm{d} s .
$$

Let us assume enough regularity of the shape $\Omega$. Following the normal direction, the gradient descent vector reads

$$
\mathbf{u}=\left\{\Delta_{s} H-\frac{1}{2}\left(H-H_{0}\right)\left[4 K-H\left(H+H_{0}\right)\right]-p-\lambda H\right\} \mathbf{n} .
$$

The transformed geometry is given at time $\mathrm{t}$ by $\Omega(t)=(\mathbf{I}+t \mathbf{u})(\Omega)$, where $t$ stands for an infinitesimal time step and $\mathbf{I}$ represents the identity transformation. We obtain

$$
\mathcal{L}(\Omega(t) ; \lambda, p)=\mathcal{L}(\Omega ; \lambda, p)-\mathrm{t} \int_{\Gamma}\left\{\Delta_{s} H-\frac{1}{2}\left(H-H_{0}\right)\left[4 K-H\left(H+H_{0}\right)\right]-p-\lambda H\right\}^{2} \mathrm{ds}+\mathcal{O}\left(\mathrm{t}^{2}\right)
$$

which obviously ensures the minimization of the objective functional. The vector $\mathbf{u}$ represents the advection field, and it depends on the level set function $\varphi$ and on the geometrical parameters $H, K$ and $\mathbf{n}$. These quantities are initially set on the membrane $\Gamma$. However, they should be encoded in terms of varphi, and then extended to the entire computational domain $\Lambda$. Collecting the elements above, we obtain the following problem:

$\mathcal{P}:$ find $\varphi, p$ and $\lambda$ such that

$$
\begin{aligned}
& \frac{\partial \varphi}{\partial t}+\left[\Delta_{s} H-\frac{1}{2}\left(H-H_{0}\right)\left[4 K-H\left(H+H_{0}\right)\right]-p-\lambda H\right] \mathbf{n} \cdot \nabla \varphi=0, \quad \text { in }(0, T) \times \Lambda \\
& \int_{\Omega} \mathrm{d} x=V_{0}, \quad \text { in }(0, T)
\end{aligned}
$$




$$
\int_{\partial \Omega} \mathrm{d} s=A_{0}, \quad \text { in }(0, T)
$$

Initial data and suitable boundary conditions are provided:

$$
\begin{aligned}
\varphi(0, \cdot)=\varphi_{0}(\cdot), & \text { in } \Lambda, \\
\varphi=\varphi_{\partial \Lambda}, & \text { on }(0, T) \times \Sigma_{-}, \\
\partial_{\nu} \varphi=0, & \text { on }(0, T) \times \partial \Lambda,
\end{aligned}
$$

where $\Sigma_{-}$represents the upstream boundary, or inflow, and $\boldsymbol{\nu}$ denotes the outward unit normal vector on the boundary $\partial \Lambda$.

\subsection{Variational formulation}

We present in this section the variational formulation of the problem (2.8a-2.8b-2.8c). At each time $t$, we assume enough regularity of the shape $\Omega$. To encode the vector of the gradient descent in terms of the level set $\varphi$, we need the following equality

$$
H\left(H^{2}-4 K\right)=2 H \nabla \cdot\left(\frac{\nabla_{s}|\nabla \varphi|}{|\nabla \varphi|}\right)-\nabla \cdot\left(H^{2} \mathbf{n}\right) .
$$

Indeed, by using the Einstein sum rule for repeated indices, we have

$\nabla \mathbf{n}: \nabla \mathbf{n}^{T}=\partial_{j} \boldsymbol{n}_{i} \partial_{i} \boldsymbol{n}_{j}=\partial_{j}\left(\boldsymbol{n}_{i} \partial_{i} \boldsymbol{n}_{j}\right)-\boldsymbol{n}_{i} \partial_{i}\left(\partial_{j} \boldsymbol{n}_{j}\right)=\nabla \cdot[(\mathbf{n} \cdot \nabla) \mathbf{n}]-\mathbf{n} \cdot \nabla H=\nabla \cdot((\mathbf{n} \cdot \nabla) \mathbf{n})-\left(\nabla \cdot(H \mathbf{n})-H^{2}\right)$.

The Gauss curvature writes $2 K=H^{2}-\nabla \mathbf{n}: \nabla \mathbf{n}^{T}$, see e.g. [47]. We obtain

$$
\begin{aligned}
H\left(H^{2}-4 K\right) & =H\left(-H^{2}+2 \nabla \mathbf{n}: \nabla \mathbf{n}^{T}\right)=2 H \nabla \cdot((\mathbf{n} \cdot \nabla) \mathbf{n})-H^{3}-2 H \mathbf{n} \cdot \nabla H \\
& =2 H \nabla \cdot((\mathbf{n} \cdot \nabla) \mathbf{n})-H^{3}-\left(\nabla \cdot\left(H^{2} \mathbf{n}\right)-H^{3}\right)=2 H \nabla \cdot((\mathbf{n} \cdot \nabla) \mathbf{n})-\nabla \cdot\left(H^{2} \mathbf{n}\right) .
\end{aligned}
$$

Moreover, the equality $\partial_{j}|\nabla \varphi|=\partial_{j} \sqrt{\nabla \varphi \cdot \nabla \varphi}=\frac{\partial_{j} \partial_{k} \varphi \partial_{k} \varphi}{|\nabla \varphi|}$, leads to

$$
(\nabla \mathbf{n})_{i j}=\partial_{j}\left(\frac{\partial_{i} \varphi}{|\nabla \varphi|}\right)=\frac{|\nabla \varphi| \partial_{j} \partial_{i} \varphi-\partial_{i} \varphi \partial_{j}|\nabla \varphi|}{|\nabla \varphi|^{2}}=\frac{1}{|\nabla \varphi|}\left(\partial_{j} \partial_{i} \varphi-\frac{\partial_{i} \varphi}{|\nabla \varphi|} \frac{\partial_{k} \varphi}{|\nabla \varphi|} \partial_{k} \partial_{j} \varphi\right) .
$$

Then we get $\nabla \mathbf{n}=\frac{1}{|\nabla \varphi|}(\mathbf{I}-\mathbf{n} \otimes \mathbf{n}) \cdot \nabla \nabla \varphi$. As we have $\nabla(\nabla \varphi \cdot \nabla \varphi)=2(\nabla \varphi \cdot \nabla) \nabla \varphi+2 \nabla \varphi \wedge \operatorname{rot}(\nabla \varphi)=$ $2 \nabla(\nabla \varphi) \cdot \nabla \varphi$, we obtain, $\nabla \nabla \varphi \cdot \mathbf{n}=\frac{\nabla(\nabla \varphi) \cdot \nabla \varphi}{|\nabla \varphi|}=\frac{1}{2} \frac{\nabla\left(|\nabla \varphi|^{2}\right)}{|\nabla \varphi|}=\nabla|\nabla \varphi|$.

Finally, we have $(\mathbf{n} \cdot \nabla) \mathbf{n}=\frac{1}{|\nabla \varphi|}((\mathbf{I}-\mathbf{n} \otimes \mathbf{n}) \cdot \nabla \nabla \varphi) . \mathbf{n}=\frac{1}{|\nabla \varphi|}(\mathbf{I}-\mathbf{n} \otimes \mathbf{n})(\nabla \nabla \varphi \cdot \mathbf{n})=\frac{\nabla_{s}|\nabla \varphi|}{|\nabla \varphi|}$. and we end with the equation (2.9). Let us consider a test function $\xi$. The variational formulation reads

$$
\int_{\Lambda} \frac{\partial \varphi}{\partial t} \xi+\int_{\Lambda}\left[2 \Delta_{s} H+H\left(H^{2}-4 K\right)\right]|\nabla \varphi| \xi=0, \quad \forall \xi \in H_{0}^{1}(\Lambda) .
$$

We denote $\varphi_{\min }$ and $\varphi_{\max }$ the maximal and minimal values of $\varphi$, respectively. By using the co-area formula, see e.g. [27], and the Green tranformation, we obtain

$$
\begin{aligned}
\int_{\Lambda}|\nabla \varphi| \Delta_{s} H \xi \mathrm{dx} & =\int_{\varphi_{\min }}^{\varphi_{\max }} \int_{\varphi(x)=z} \Delta_{s} H \xi \mathrm{dsdz} \\
& =\int_{\varphi_{\min }}^{\varphi_{\max }}(-\int_{\varphi(x)=z} \nabla_{s} H \cdot \nabla_{s} \xi \mathrm{ds}+\int_{\varphi(x)=z} \xi H \underbrace{\mathbf{n} \cdot \nabla_{s} H}_{0} \mathrm{ds}) \mathrm{dz}=-\int_{\Lambda}|\nabla \varphi| \nabla_{s} H . \nabla_{s} \xi \mathrm{dx} .
\end{aligned}
$$

The surface projection operator verify $(\mathbf{I}-\mathbf{n} \otimes \mathbf{n})^{2}=(\mathbf{I}-\mathbf{n} \otimes \mathbf{n})^{T}=(\mathbf{I}-\mathbf{n} \otimes \mathbf{n})$, and we have the equality $\int_{\Lambda}|\nabla \varphi| \Delta_{s} H \xi+\int_{\Lambda}|\nabla \varphi| \nabla_{s} H \cdot \nabla \xi=0$. Using the equation (2.9) and the homogeneous Neumann boundary condition $\partial_{\nu} \varphi=0$, we obtain

$$
\int_{\Lambda}|\nabla \varphi| H\left(H^{2}-4 K\right) \xi=2 \int_{\Lambda}|\nabla \varphi| H \nabla \cdot\left(\frac{\nabla_{s}|\nabla \varphi|}{|\nabla \varphi|}\right) \xi-\int_{\Lambda}|\nabla \varphi| \nabla \cdot\left(H^{2} \mathbf{n}\right) \xi
$$




$$
\begin{aligned}
& =-2 \int_{\Lambda} \frac{\nabla_{s}|\nabla \varphi|}{|\nabla \varphi|} \cdot \nabla(|\nabla \varphi| H \xi)+\int_{\Lambda} H^{2} \mathbf{n} \cdot \nabla(|\nabla \varphi| \xi) \\
& =\int_{\Lambda}\left(H^{2} \nabla \varphi-2 H \nabla_{s}|\nabla \varphi|\right) \cdot \nabla \xi-2 \int_{\Lambda} \frac{\nabla|\nabla \varphi|}{|\nabla \varphi|} \cdot \nabla_{s}(H|\nabla \varphi|) \xi+\int_{\Lambda} \frac{H^{2}}{|\nabla \varphi|} \nabla \varphi \cdot \nabla(|\nabla \varphi|) \xi .
\end{aligned}
$$

Using the equality $|\nabla \varphi| \nabla_{s} H+H \nabla_{s}|\nabla \varphi|=\nabla_{s}(|\nabla \varphi| H)$, the equation (2.10) reads

$$
\int_{\Lambda} \frac{\partial \varphi}{\partial t} \xi-2 \int_{\Lambda} \nabla_{s}(H|\nabla \varphi|) \cdot \nabla \xi+\int_{\Lambda} H^{2} \nabla \varphi \cdot \nabla \xi-2 \int_{\Lambda} \frac{\nabla|\nabla \varphi|}{|\nabla \varphi|} \cdot \nabla_{s}(H|\nabla \varphi|) \xi+\int_{\Lambda} \frac{H^{2}}{|\nabla \varphi|} \nabla \varphi \cdot \nabla(|\nabla \varphi|) \xi=0 .
$$

To facilitate the numerical discretization, we write a mixed problem to decrease the derivation order of $\varphi$ in the equation 2.11, Let us consider the mixed variable $\psi=-H|\nabla \varphi|$. The equation (2.11) becomes

$$
\begin{aligned}
& \int_{\Lambda} \frac{\partial \varphi}{\partial t} \xi+\int_{\Lambda} \nabla_{s}(\psi) \cdot \nabla \xi+\frac{1}{2} \int_{\Lambda} \frac{\psi^{2}}{|\nabla \varphi|^{2}} \nabla \varphi \cdot \nabla \xi+\int_{\Lambda} \frac{\nabla|\nabla \varphi|}{|\nabla \varphi|} \cdot \nabla_{s}(\psi) \xi \\
& +\frac{1}{2} \int_{\Lambda} \frac{\psi^{2}}{|\nabla \varphi|^{3}} \nabla \varphi \cdot \nabla(|\nabla \varphi|) \xi-\int_{\Lambda} p|\nabla \varphi| \xi+\int_{\Lambda} \lambda \psi \xi=0, \quad \forall \xi \in H_{0}^{1}(\Lambda) \\
& \int_{\Lambda} \frac{\psi}{|\nabla \varphi|} \zeta-\int_{\Lambda} \frac{1}{|\nabla \varphi|} \nabla \varphi \cdot \nabla \zeta=0, \quad \forall \zeta \in H^{1}(\Lambda) .
\end{aligned}
$$

From a numerical point of view, we give a particular attention to the skeleton of the level set function. In fact, the outward normal vector $\mathbf{n}$ becomes discontinuous when crossing this area, and a subdifferential of the level set function exists. By considering some elementary shapes, figure 2 depicts these particular areas, in which we observe that the norm of the gradient $|\nabla \varphi|$ has very small values, i.e. around the machine precision. Therefore, after the numerical discretization of (2.12 2.13$)$, the assembled matrix of the linear system may become singular if some nodes are located in the skeleton. To address this difficulty, a regularization technique may be used in the neighborhood of the skeleton. In the present work, we decided to avoid the division by $|\nabla \varphi|$ in the weak formulation (2.12) 2.13). Let us consider two integers $(k, l) \in \mathbb{N}^{2}$, and we introduce two test functions $(\widetilde{\xi}, \widetilde{\zeta}) \in H_{0}^{1}(\Lambda) \times H^{1}(\Lambda)$ such that $\xi=|\nabla \varphi|^{l} \widetilde{\xi}$ and $\zeta=|\nabla \varphi|^{k} \widetilde{\zeta}$. The variational formulation (2.12|2.13) writes

$$
\begin{aligned}
& \int_{\Lambda}|\nabla \varphi|^{l} \partial_{t} \varphi \widetilde{\xi}+\int_{\Lambda}|\nabla \varphi|^{l} \nabla_{s}(\psi) \cdot \nabla \widetilde{\xi}+\frac{1}{2} \int_{\Lambda}|\nabla \varphi|^{l-2} \psi^{2} \nabla \varphi \cdot \nabla \widetilde{\xi}-\int_{\Lambda} p|\nabla \varphi|^{l+1} \widetilde{\xi} \\
& +\int_{\Lambda}(l+1)|\nabla \varphi|^{l-1} \nabla|\nabla \varphi| \cdot \nabla_{s}(\psi) \widetilde{\xi}+\frac{1}{2} \int_{\Lambda}(l+1)|\nabla \varphi|^{l-3} \psi^{2} \nabla \varphi \cdot \nabla(|\nabla \varphi|) \widetilde{\xi}+\int_{\Lambda} \lambda \psi|\nabla \varphi|^{l} \widetilde{\xi}=0, \\
& \int_{\Lambda}|\nabla \varphi|^{k-1} \psi \widetilde{\zeta}-\int_{\Lambda}|\nabla \varphi|^{k-1} \nabla \varphi \cdot \nabla \widetilde{\zeta}=\int_{\Lambda} k|\nabla \varphi|^{k-2} \nabla \varphi \cdot \nabla|\nabla \varphi| \widetilde{\zeta} .
\end{aligned}
$$

By setting $l=3$ and $k=2$, we can avoid the division by $|\nabla \varphi|$. In what follows, we will drop the tilde symbol to make reading easier. Let us introduce the mixed variable $\gamma$ given by

$$
\boldsymbol{\gamma}=\nabla|\nabla \varphi| \in \mathrm{H}_{0}(\operatorname{div}, \Lambda):=\left\{\boldsymbol{\tau} \in\left(L^{2}(\Lambda)\right)^{d}: \nabla \cdot \boldsymbol{\tau} \in L^{2}(\Lambda) \text { and } \boldsymbol{\tau} \cdot \boldsymbol{\nu}_{\partial \Lambda}=0 \text { on } \partial \Lambda\right\}
$$

Finally, the variational formulation reads:

given $\varphi_{\partial \Lambda} \in \mathcal{C}^{1}\left([0, T] ; L^{2}(\Lambda)\right) \cap L^{2}\left((0, T) ; H^{1}(\Lambda) \cap W^{1, \infty}(\Lambda)\right)$ and $\varphi_{0} \in L^{2}(\Lambda)$,

find $\mathbf{n} \in L^{2}\left((0, T) ; L^{\infty}(\Lambda)^{d}\right), \gamma \in L^{2}\left((0, T) ; H_{0}(d i v, \Lambda) \cap L^{\infty}(\Lambda)^{d}\right), \psi \in L^{2}\left((0, T) ; H^{1}(\Lambda) \cap L^{\infty}(\Lambda)\right)$, and $\varphi$ with $\varphi-\varphi_{\partial \Lambda} \in \mathcal{C}^{1}\left([0, T] ; L^{2}(\Lambda)\right) \cap L^{2}\left((0, T) ; H_{0}^{1}(\Lambda) \cap W^{1, \infty}(\Lambda)\right)$ such that

$$
\begin{aligned}
& \int_{\Lambda}|\nabla \varphi| \mathbf{n} \cdot \varsigma=\int_{\Lambda} \nabla \varphi \cdot \varsigma, \quad \forall \varsigma \in\left(L^{2}(\Lambda)\right)^{d} \\
& \int_{\Lambda} \gamma \cdot \boldsymbol{\tau}=-\int_{\Lambda}|\nabla \varphi| \nabla \cdot \boldsymbol{\tau}, \quad \forall \boldsymbol{\tau} \in H_{0}(\operatorname{div}, \Lambda) \\
& \int_{\Lambda}|\nabla \varphi| \psi \xi-\int_{\Lambda}|\nabla \varphi| \nabla \varphi \cdot \nabla \xi=2 \int_{\Lambda}(\nabla \varphi \cdot \gamma) \xi, \quad \forall \xi \in H^{1}(\Lambda) \\
& \int_{\Lambda}|\nabla \varphi|^{3} \nabla_{s} \psi \cdot \nabla \zeta+\int_{\Lambda}|\nabla \varphi|^{3} \partial_{t} \varphi \zeta+\frac{1}{2} \int_{\Lambda}|\nabla \varphi| \psi^{2} \nabla \varphi \cdot \nabla \zeta \\
& -\int_{\Lambda} p|\nabla \varphi|^{4} \zeta+4 \int_{\Lambda}|\nabla \varphi|^{2}\left(\nabla_{s} \psi \cdot \gamma\right) \zeta+2 \int_{\Lambda} \psi^{2}(\nabla \varphi \cdot \gamma) \zeta+\int_{\Lambda} \lambda \psi|\nabla \varphi|^{3} \zeta=0, \quad \forall \zeta \in \mathrm{H}_{0}^{1}(\Lambda) .
\end{aligned}
$$



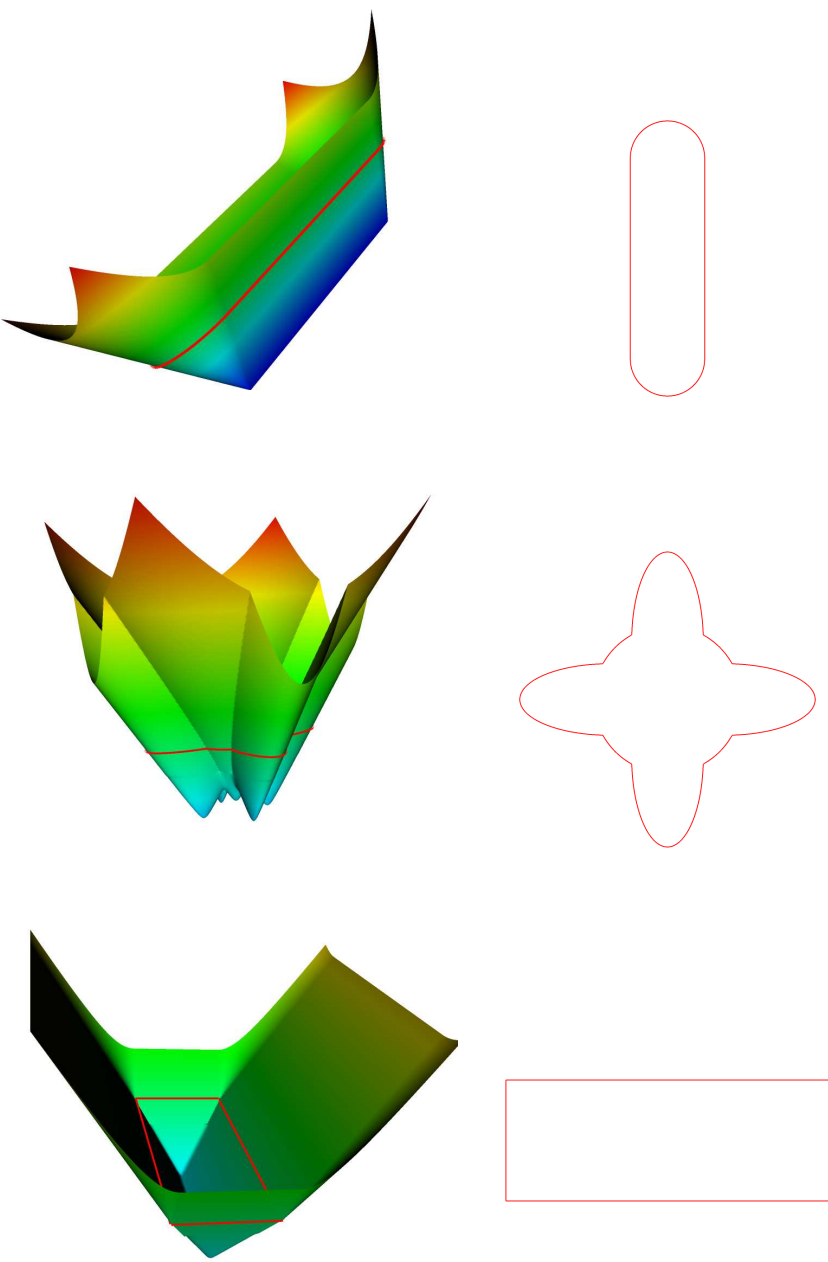
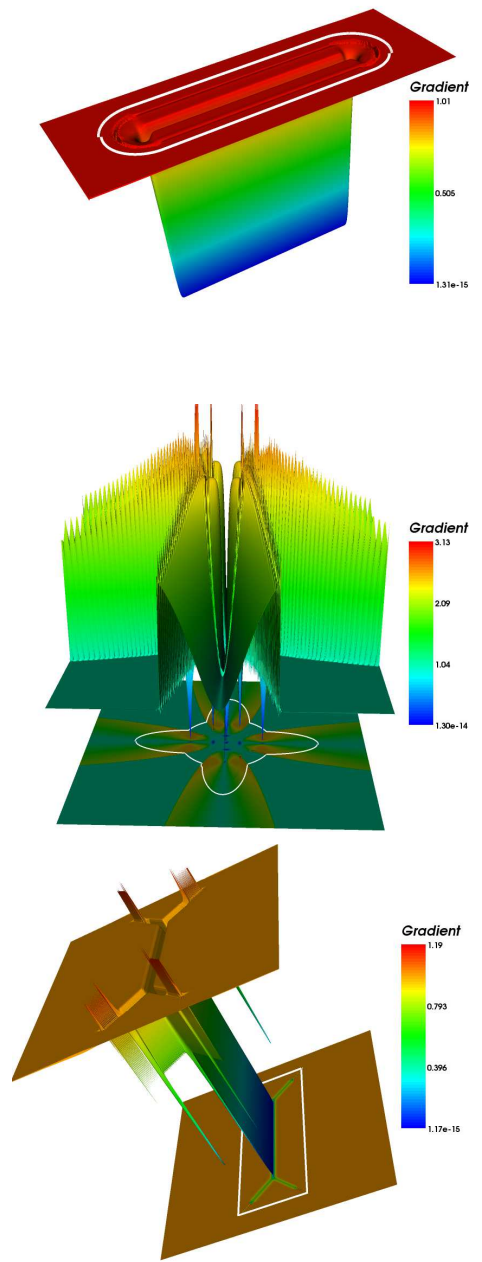

Figure 2: Singularities in the skeleton for three different shapes. (left) The function $\varphi$ is plotted in elevation;the zero-level set is plotted in red. (middle) The zero-level set $\Gamma$. (right) The gradient $|\nabla \varphi|$ and its projection in the horizontal plan.

\subsection{Refinement and mesh adaptation technique}

Our approach bases on the work of Borouchaki et al. [10, 9, 30], and we use the bidirectional anisotropic mesh generator BAMG developed by F. Hecht et al. [38], see also [40, 41]. We first summarize the principles on which this generator is based. Let us consider a governing field $\Xi$ to be suitably computed from the solution of the discretized problem (2.14]2.15]2.16 2.17). The choice of this field assumes that we have some knowledge of a particular aspect of the solution. Then, the mesh is adapted to the computation of $\Xi$ such that its interpolation error becomes uniformly distributed. It means that this error becomes constant over all the mesh triangles in the directions of maximal and minimal stretching, while the maximal and minimal directions of stretching have to be adjusted to the directions of maximal and minimal error. Therefore, our approach consists in the choice of a particular metric that is specific to our time-dependent shape optimization problem.

We consider a partition $\mathscr{T}_{h}$ of $\Lambda$ at time $t$ consisting of geometrically conforming open simplicial elements $K$, i.e. triangles in the two-dimensional case, such that $\bar{\Lambda}=\cup_{K \in \mathscr{T}_{h}}$. In this paragraph, the subscribes $\xi$ and $x$ stand for the reference and the deformed configurations, respectively. Let $X_{K}$ be the affine transformation which maps the reference triangle $K_{\xi}$ into $K_{x}$ as depicted in Fig. 3 .

$$
\begin{aligned}
X_{K}: & K_{\xi} \\
\boldsymbol{\xi} & \longmapsto \\
& \longmapsto \mathbf{x}=M_{K} \boldsymbol{\xi}+\mathbf{t}_{K},
\end{aligned}
$$

where $M_{K}$ represents the Jacobian of the transformation $X_{K}$, and $\mathbf{t}_{K}$ is a translation motion. Since the element $\mathrm{K}$ of the mesh is not a flat triangle, the matrix $M_{K}$ is asymmetric and invertible. Hence, the matrix $M_{K}$ admits a 


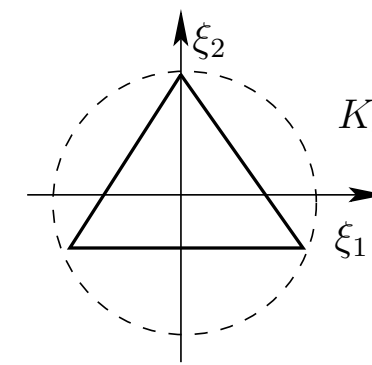

reference configuration

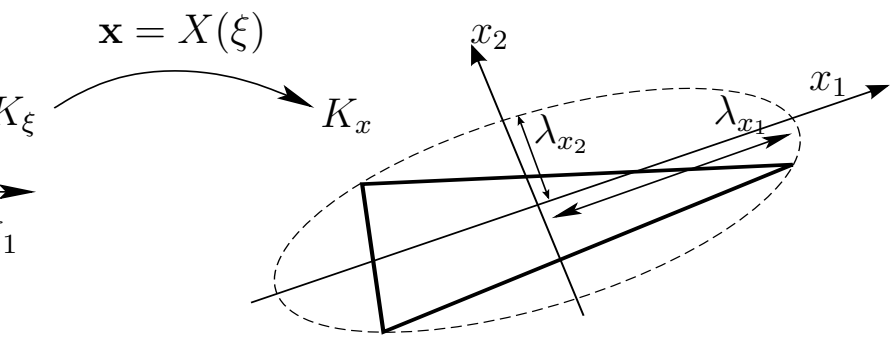

actual configuration

Figure 3: Transformation from the reference element $K_{\xi}$ to the triangle $K_{x}$ in the actual configuration.
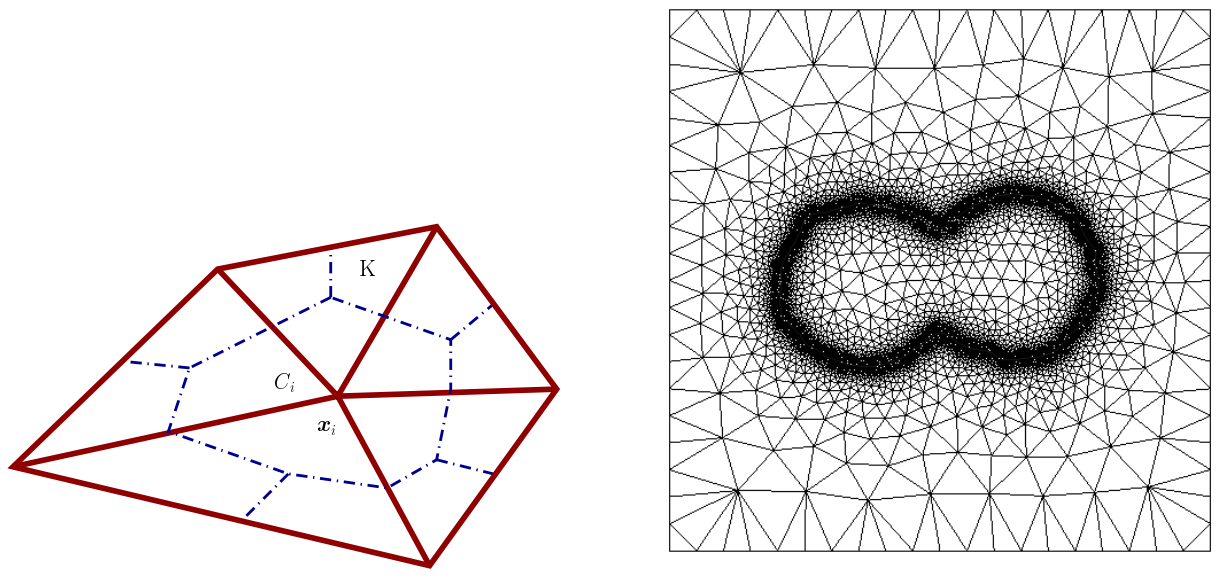

Figure 4: (left) The finite volume cell $C_{i}$, centered around the vertex $x_{i} \in \mathcal{T}_{h}$, joins the barycenters of the triangles and the middle points of edges passing through $\boldsymbol{x}_{i}$. (right) Adapted mesh around the RBC's membrane with 10.732 triangles and 5.407 nodes.

singular value decomposition, denoted simply by SVD, see e.g. 35, 36, 34]. The SVD expresses the matrix $M_{K}$ as a product: $M_{K}=R_{K}^{T} \Lambda_{K} P_{K}$, where $R_{K}$ and $P_{K}$ are orthogonal and $\Lambda_{K}$ is a diagonal matrix with positive entries which are known as the singular values of $M_{K}$. Several possible choices for the reference triangle $K_{\xi}$ exist, and it is quit common to choose $K_{\xi}=\left\{\left(\xi_{1}, \xi_{2}\right) \in \mathbb{R}^{2}: \xi_{1}>0, \xi_{2}>0\right.$ and $\left.\xi_{1}+\xi_{2}<1\right\}$. The equilateral triangle inscribed in the unit circle, i.e. with unit radius, is generally considered for purposes of mesh generation, see 28]. Since the coordinates in the reference configuration verify $\boldsymbol{\xi}=M_{K}^{-1}\left(\boldsymbol{x}-\mathbf{t}_{K}\right)$, the unit circle equation given by $\boldsymbol{\xi}^{T} \boldsymbol{\xi}=1$ writes

$$
1=\left(\boldsymbol{x}-\mathbf{t}_{K}\right)^{T} M_{K}^{-T} M_{K}^{-1}\left(\boldsymbol{x}-\mathbf{t}_{K}\right)=\left(\boldsymbol{x}-\mathbf{t}_{K}\right)^{T} R_{K}^{T} \Lambda_{K}^{-2} R_{K}\left(\boldsymbol{x}-\mathbf{t}_{K}\right),
$$

which represents the equation of an ellipse containing $K_{x}$ as depicted in Fig. 3 (right). The governing field $\Xi$ is considered, and the metric tensor is computed from the Hessian matrix of $\Xi$, see [38]. We adapt the mesh with respect to the computation of $\Xi$, and the purpose is to decrease the interpolation error. Piecewise linear and continuous functions are used to approximate the field $\Xi$ over each mesh element $K \in \mathcal{T}_{h}$, and the error of interpolation in the direction $\mathbf{v} \in \mathbb{R}^{2}$ is estimated by:

$$
e_{K, \mathbf{v}}=h_{K, \mathbf{v}}^{2}\left|\frac{\partial^{2} \chi}{\partial \mathbf{v}^{2}}\right|=h_{K, \mathbf{v}}^{2}\left|\mathbf{v}^{T}(\nabla \nabla \Xi) \mathbf{v}\right| \quad \text { in } K
$$

The parameter $h_{K, \mathbf{v}}$ represents the length of $K$ in the direction $\mathbf{v}$. The matrix $\nabla \nabla \Xi$ denotes a piecewise linear approximation of the Hessian of the governing field $\Xi$. Let us consider the eigenvalues $\lambda_{x_{1}}$ and $\lambda_{x_{2}}$ of the Hessian $\nabla \nabla \Xi$. The corresponding eigenvectors are denoted $\boldsymbol{v}_{1}$ and $\boldsymbol{v}_{2}$, respectively. Therefore, the mesh generator tool will adapt the mesh by shrinking the elements $K$ in both directions $\boldsymbol{v}_{1}$ and $\boldsymbol{v}_{2}$. The directional sizes $h_{K, \mathbf{v}_{1}}$ and $h_{K, \mathbf{v}_{2}}$ are then adjusted to equidistribute the interpolation error in the mesh element $K$, and the mesh generator refine then the initial mesh in regions where more accuracy is needed. Regarding the choice of the governing field $\Xi$, it represents an important step in this procedure of refinement, since it allows to emphasize on regions where 
accurate numerical computations are needed. For any time $t^{n}$, we need to refine the mesh especially in the vicinity of the membrane of the RBC, wherein several quantities such as the curvature, the normal vector and the surface operators must be computed with high accuracy. We decide to set the governing field $\Xi=\delta_{\varepsilon}\left(\varphi^{n}\right)+\delta_{\varepsilon}\left(\varphi^{n-1}\right)$. In cases where big deformations happens, we include also an other term that describes a prediction of the solution at time $t^{n+1}$. At each node $\boldsymbol{x}_{i}$ in the mesh $\mathcal{T}_{h}$, the regularization parameter $\varepsilon$ is proportional to the local value of the mesh size $\varepsilon\left(\boldsymbol{x}_{i}\right)=2 h_{\mid C_{i}}=2 \sqrt{2}$ meas $\left(\mathrm{C}_{\mathrm{i}}\right)^{\frac{1}{2}}$, where $C_{i}$ represents the finite element cell centered in $\boldsymbol{x}_{i}$, see Fig 4 (left). Fig. 4 shows the adapted mesh around the RBC's membrane. For all mesh triangles $K$, the mesh is characterized by $\mathcal{M}_{1} \in[0.45,1]$ and $\mathcal{M}_{2} \in[0.34,0.9]$, where $\mathcal{M}_{1}$ represents the ratio between the inscribed radius and the circumscribed radius of the mesh element $\mathrm{K}$, and $\mathcal{M}_{2}$ represents the ratio between the minimal edge length and the maximal edge length in the element $K$, see [31] for the detailed description of these parameters.

\section{Numerical approximation}

\subsection{Time discretization}

To discretize the problem $(2.14] 2.15] 2.16+2.17)$ in time, we divide the time interval $[0, T]$ into $N$ subintervals $\left[t_{n}, t_{n+1}\right)$ of constant step $\Delta t$ where $n=0, \cdots, N-1$. For any $n \geq 1$, the unknowns $\psi^{n}$ and $\varphi^{n}$ at $t^{n}$ are computed recursively. The time discretization is performed by using the second-order forward Euler approximation. For a given function $\mathcal{F} \in \mathcal{C}^{1}([0, T])$ and $t \in(0, T)$, we have $\mathcal{F}^{\prime}(t)=\frac{3 \mathcal{F}(t)-4 \mathcal{F}(t-\Delta t)+\mathcal{F}(t-2 \Delta t)}{2 \Delta t}+\frac{\mathcal{F}^{(3)}(t)}{3} \Delta t^{2}$. The scheme approximation writes

$$
\mathcal{F}^{\prime}(t) \approx \frac{3}{2} \frac{\mathcal{F}^{n+1}-\mathcal{F}^{n}}{\Delta t} \quad \text { if } n=1 \quad \text { and } \quad \mathcal{F}^{\prime}(t) \approx \frac{3 \mathcal{F}^{n+1}-4 \mathcal{F}^{n}+\mathcal{F}^{n-1}}{2 \Delta t} \quad \text { if } n>1 .
$$

By using a second order schema in time, one can expect that the error follows the evolution $\mathcal{O}\left(h^{k}\right)+\mathcal{O}\left(\triangle t^{2}\right)$ where $k$ represents the degree of the polynomial finite element discretization in space. The theoretical analysis regarding the error estimation is preserved for further work. We introduce the weighted multi-linear forms

$$
\begin{array}{ll}
m(w, \xi, \zeta)=\int_{\Lambda} w \xi \zeta & \text { for all } w \in L^{\infty}(\Lambda), \xi \in L^{2}(\Lambda) \text { and } \zeta \in L^{2}(\Lambda) \\
a(\mathbf{T}, \xi, \zeta)=\int_{\Lambda}(\mathbf{T} \nabla \xi) \cdot \nabla \zeta & \text { for all } \mathbf{T} \in\left(L^{\infty}(\Lambda)\right)^{d \times d}, \xi \in H^{1}(\Lambda) \text { and } \zeta \in H^{1}(\Lambda) .
\end{array}
$$

A semi-implicite scheme is used for the numerical approximation in time of the equations (2.16) and (2.17). The variational problem reads $\left(p^{n}, \lambda^{n}\right) \in \mathbb{R}^{2}$

$\mathcal{P}_{t}:$ given $\varphi_{\partial \Lambda}^{n} \in H^{1}(\Lambda) \cap W^{1, \infty}(\Lambda), \varphi_{0}^{0}=\varphi_{0}^{-1} \in L^{2}(\Lambda), \mathbf{n}^{n} \in L^{\infty}(\Lambda)^{d}, \gamma^{n} \in H_{0}(\operatorname{div}, \Lambda) \cap L^{\infty}(\Lambda)^{d}$ and find $\psi^{n+1} \in H^{1}(\Lambda) \cap L^{\infty}(\Lambda)$ and $\varphi^{n+1}$ with $\varphi^{n+1}-\varphi_{\partial \Lambda}^{n} \in H_{0}^{1}(\Lambda) \cap W^{1, \infty}(\Lambda)$ such that

$$
\begin{aligned}
& m\left(\left|\nabla \varphi^{n}\right|, \psi^{n+1}, \xi\right)-a\left(\left|\nabla \varphi^{n}\right| \mathbf{I}, \varphi^{n+1}, \xi\right)=2 m\left(\nabla \varphi^{n} \cdot \gamma^{n}, 1, \xi\right) \\
& a\left(\left|\nabla \varphi^{n}\right|^{3}\left(\mathbf{I}-\mathbf{n}^{n} \otimes \mathbf{n}^{n}\right), \psi^{n+1}, \zeta\right)+\frac{3}{2 \triangle t} m\left(\left|\nabla \varphi^{n}\right|^{3}, \varphi^{n+1}, \zeta\right)+\frac{1}{2} a\left(\left|\nabla \varphi^{n}\right|\left(\psi^{n}\right)^{2} \mathbf{I}, \varphi^{n+1}, \zeta\right)= \\
& \frac{1}{2 \triangle t} m\left(\left|\nabla \varphi^{n}\right|^{3}\left(4 \varphi^{n}-\varphi^{n-1}\right), 1, \zeta\right)-m\left(\lambda^{n} \psi^{n}\left|\nabla \varphi^{n}\right|^{3}, 1, \zeta\right) \\
& -m\left(4\left|\nabla \varphi^{n}\right|^{2}\left(\mathbf{I}-\mathbf{n}^{n} \otimes \mathbf{n}^{n}\right) \nabla \psi^{n} \cdot \gamma^{n}+2\left(\psi^{n}\right)^{2}\left(\nabla \varphi^{n} \cdot \gamma^{n}\right)-p^{n}\left|\nabla \varphi^{n}\right|^{4}, 1, \zeta\right)
\end{aligned}
$$

for all $\xi \in H^{1}(\Lambda)$ and $\forall \zeta \in H_{0}^{1}(\Lambda)$.

\subsection{Algorithm for the saddle point problem}

To compute the saddle points of the Lagrangian $\mathcal{L}(\Omega ; p, \lambda)$, we use an Uzawa-type algorithm. This algorithm was originally introduced by Arrow et al. 3] in the context of concave programming in order to solve saddle point problems, see [29, 32, 33] for a variety of Uzawa-type methods. This algorithm performs two steps: it first minimizes the Lagrangian with respect to the shape, then the Lagrange multipliers are computed by minimizing 


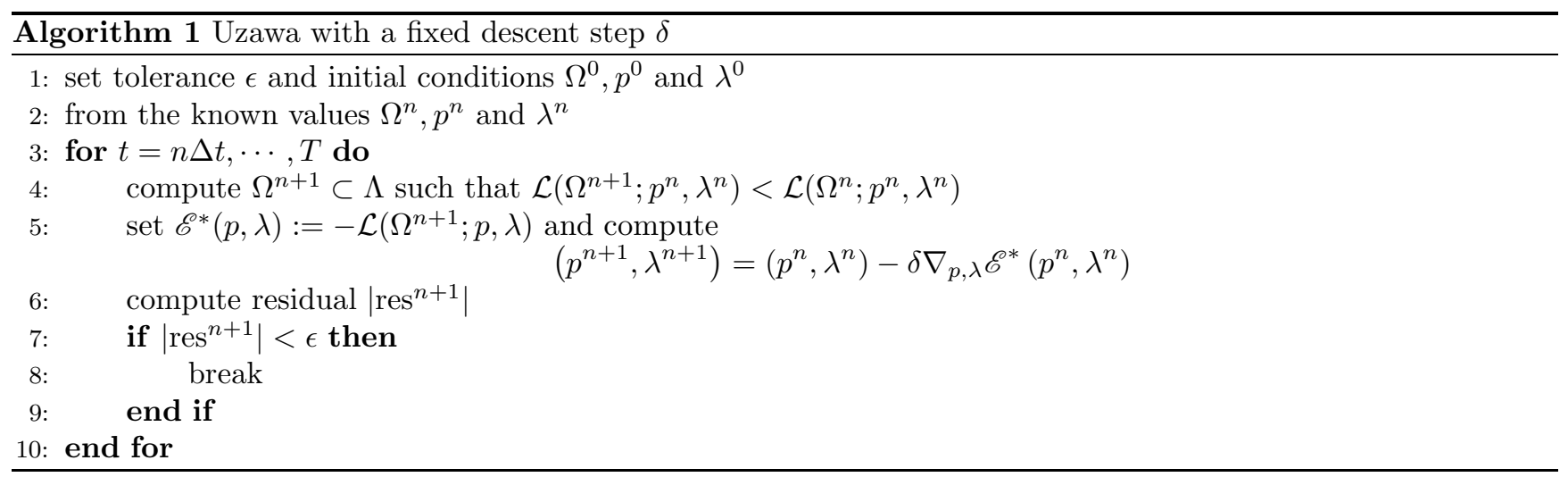

the dual energy $\mathscr{E}^{*}(p, \lambda)$, as detailed in Algorithm 1, In fact, the Uzawa method represents a descent method in the direction opposite to the gradient of $\mathscr{E} *(p, \lambda)$ with a constant step length $\delta$, and we assume $\delta$ small enough to ensure the convergence. As we will show afterwards, a natural choice of the stopping criterion should depend on the residual of the approximate solutions $\psi$ and $\varphi$. We compute at each time $t^{n}$ the two residuals $\operatorname{res}_{I I}^{n}$ and res ${ }_{I}^{n}$ of the equations (3.1a) and (3.1b), respectively. Let us define the global residual at time $t^{n}$ by $\left|\operatorname{res}^{n}\right|=\sqrt{\left|\operatorname{res}_{I}^{n}\right|^{2}+\left|\operatorname{res}_{I I}^{n}\right|^{2}}$ where $|\cdot|$ denotes an appropriate norm for the residual, see section 3.3 for further details about the numerical computation of the residuals. By using a fixed descent step $\delta$, the Uzawa algorithm does not preserve the volume and the surface area at each iteration. The two constraints will be verified only on the convergence. However, the two constraints may be verified if the Uzawa algorithm is used with an optimal descent step. In such case, the exact Lagrange multipliers should be computed at each iteration and the algorithm has more stabily and effectiveness. The Uzawa algorithm with the exact descent step is shown in Algorithm 2 .

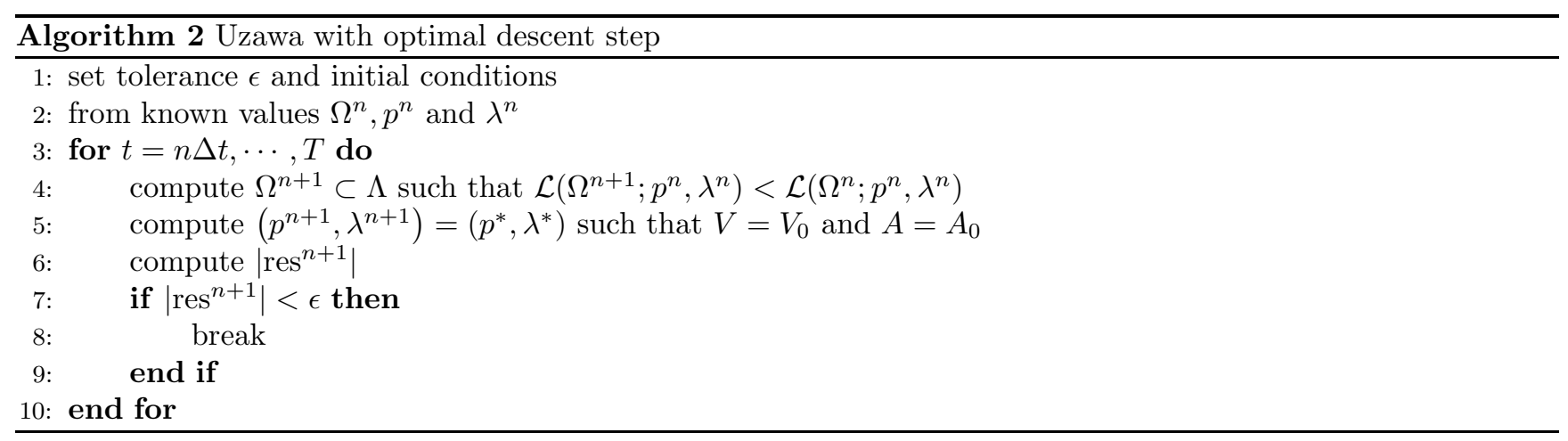

Computation of the optimal Lagrange multipliers. In this section, we proceed to compute the exact values of the Lagrange multipliers $p^{*}$ and $\lambda^{*}$, and we solve the problem $\mathcal{P}(2.8 \mathrm{a}, 2.8 \mathrm{~b}, 2.8 \mathrm{c})$. We have previously shown that $(\mathbf{n} \cdot \nabla) \mathbf{n}=\frac{\nabla_{s}|\nabla \varphi|}{|\nabla \varphi|}$ and $H\left(H^{2}-4 K\right)=2 H \nabla \cdot((\mathbf{n} \cdot \nabla) \mathbf{n})-\nabla \cdot\left(H^{2} \mathbf{n}\right)$. Then the following equation is satisfied $\frac{1}{2} H\left(H^{2}-4 K\right)=H \nabla \cdot\left[\frac{\nabla_{s}|\nabla \varphi|}{|\nabla \varphi|}\right]-\frac{1}{2} \nabla \cdot\left(H^{2} \mathbf{n}\right)$. The problem $\mathcal{P}(2.8 \mathrm{a}, 2.8 \mathrm{~b}-2.8 \mathrm{c})$ writes

$$
\begin{aligned}
& \frac{\partial \varphi}{\partial t}+\left[\Delta_{s} H+H \nabla \cdot\left[\frac{\nabla_{s}|\nabla \varphi|}{|\nabla \varphi|}\right]-\frac{1}{2} \nabla \cdot\left(H^{2} \mathbf{n}\right)-p-\lambda H\right]|\nabla \varphi|=0 \quad \text { in }(0, T) \times \Lambda \\
& \frac{d}{d t} \int_{\Lambda} \mathscr{H}(\varphi)=0 \quad \text { in }(0, T) \\
& \frac{d}{d t} \int_{\Gamma} 1=0 \quad \text { in }(0, T) .
\end{aligned}
$$


To get the expressions of the Lagrange multipliers, we start with the equation (3.4). Thanks to the Reynolds formula, we are able to simplify this equation and we have successively

$$
\begin{aligned}
& \frac{d}{d t} \int_{\Gamma} 1=0 \Leftrightarrow \int_{\Gamma} \nabla_{s} \cdot \mathbf{u}=0 \Leftrightarrow \int_{\Gamma} H \mathbf{u} \cdot \mathbf{n}=0 \Leftrightarrow \\
& p \int_{\Gamma} H+\lambda \int_{\partial \Omega} H^{2}=\int_{\Gamma} H \Delta_{s} H+\int_{\Gamma} H^{2} \nabla \cdot\left(\frac{\nabla_{s}|\nabla \varphi|}{|\nabla \varphi|}\right)-\frac{1}{2} \int_{\Gamma} H \nabla \cdot\left(H^{2} \mathbf{n}\right) .
\end{aligned}
$$

Regarding the equation (3.3), we obtain, using (3.3)

$$
\begin{aligned}
& \frac{d}{d t} \int_{\Lambda} \mathscr{H}(\varphi)=0 \Leftrightarrow \int_{\Lambda} \frac{\partial \varphi}{\partial t} \delta(\varphi)=0 \Leftrightarrow \int_{\Gamma} \frac{\partial \varphi}{\partial t} \frac{1}{|\nabla \varphi|}=0 \Leftrightarrow \\
& p \int_{\Gamma} 1+\lambda \int_{\partial \Omega} H=\int_{\Gamma} \Delta_{s} H+\int_{\Gamma} H \nabla \cdot\left[\frac{\nabla_{s}|\nabla \varphi|}{|\nabla \varphi|}\right]-\frac{1}{2} \int_{\Gamma} \nabla \cdot\left(H^{2} \mathbf{n}\right) .
\end{aligned}
$$

The Green's formula involving surface integrals reads $\int_{\Gamma} \Delta_{s} H=-\int_{\Gamma} \nabla_{s} 1 \cdot \nabla_{s} H+\int_{\Gamma} H \mathbf{n} . \nabla_{s} H=0$. Then, using formula of integration by parts on surfaces $\int_{\Gamma} H \Delta_{s} H=-\int_{\Gamma}\left|\nabla_{s} H\right|^{2}+\int_{\Gamma} H^{2} \mathbf{n} \cdot \nabla_{s} H=-\int_{\Gamma}\left|\nabla_{s} H\right|^{2}$ and collecting the elements above, we obtain the system

$$
\begin{aligned}
& p \int_{\Gamma} H+\lambda \int_{\Gamma} H^{2}=-\int_{\Gamma}\left|\nabla_{s} H\right|^{2}+\int_{\Gamma} H^{2} \nabla \cdot\left[\frac{\nabla_{s}|\nabla \varphi|}{|\nabla \varphi|}\right]-\frac{1}{2} \int_{\Gamma} H \nabla \cdot\left(H^{2} \mathbf{n}\right) \\
& p \int_{\Gamma} 1+\lambda \int_{\Gamma} H=\int_{\Gamma} H \nabla \cdot\left[\frac{\nabla_{s}|\nabla \varphi|}{|\nabla \varphi|}\right]-\frac{1}{2} \int_{\Gamma} \nabla \cdot\left(H^{2} \mathbf{n}\right) .
\end{aligned}
$$

Finally, the Lagrange multipliers $p^{*}$ and $\lambda^{*}$ are given by

$$
\begin{aligned}
\lambda^{*}= & \frac{\int_{\Gamma} 1 \times \int_{\Gamma} H^{2} \nabla \cdot\left(\frac{\nabla_{s}|\nabla \varphi|}{|\nabla \varphi|}\right)-\left|\nabla_{s} H\right|^{2}-\frac{H}{2} \nabla \cdot\left(H^{2} \mathbf{n}\right)}{\int_{\Gamma} 1 \int_{\Gamma} H^{2}-\left(\int_{\Gamma} H\right)^{2}}-\frac{\int_{\Gamma} H \times \int_{\Gamma} H \nabla \cdot\left(\frac{\nabla_{s}|\nabla \varphi|}{|\nabla \varphi|}\right)-\frac{\nabla \cdot\left(H^{2} \mathbf{n}\right)}{2}}{\int_{\Gamma} 1 \int_{\Gamma} H^{2}-\left(\int_{\Gamma} H\right)^{2}} \\
p^{*}= & \frac{\int_{\Gamma} H \nabla \cdot\left(\frac{\nabla_{s}|\nabla \varphi|}{|\nabla \varphi|}\right)-\frac{1}{2} \int_{\Gamma} \nabla \cdot\left(H^{2} \mathbf{n}\right)-\lambda \int_{\Gamma} H}{\int_{\Gamma} 1} .
\end{aligned}
$$

Remark that the expressions of the Lagrange multipliers become simpler in cases where $\varphi$ is a signed distance function. In such cases, some integrals vanish and we have $\int_{\Gamma} H \nabla \cdot\left(\frac{\nabla_{s}|\nabla \varphi|}{|\nabla \varphi|}\right)=\int_{\Gamma} H^{2} \nabla \cdot\left(\frac{\nabla_{s}|\nabla \varphi|}{|\nabla \varphi|}\right)=0$. However, we assume that we consider only topologies where the zero-level set $\Gamma$ does not touch the skeleton of the function $\varphi$. Since the norm of the gradient $|\nabla \varphi|$ is evaluated only in the vicinity of $\Gamma$ where, thanks to the redistancing step, the level set function is close to the signed distance function. Therefore, the division by $|\nabla \varphi|$ in the equations (3.6) and (3.5) does not induce numerical singularities.

\subsection{Space discretization by finite elements}

We proceed with the space discretization of the problem $\mathcal{P}_{t}$ 3.1a 3.1b). We consider a partition $\mathscr{T}_{h}$ of $\Lambda$ consisting of geometrically conforming open simplicial elements $K$ (triangles for $d=2$ ), such that $\bar{\Lambda}=\cup_{K \in \mathscr{T}_{h}}$. The mesh parameter is $h=\max _{K \in \mathscr{T}_{h}} \operatorname{diam}(K)$ if the mesh is regular. However, the mesh discretization parameter is given by $h=h_{\mathrm{eq}}$ if the mesh is adapted. The equivalent space discretization step is given by $h_{e q}=1 / \sqrt{N_{n}}$, where $N_{n}$ represents the number of nodes in the adapted mesh. A piecewise continuous finite element approximation is considered for the discretization of the level set $\varphi$ and the mixed variable $\psi$. Let us denote by $\varphi_{h}^{n}$ and $\psi_{h}^{n}$ the spatial approximation of $\varphi^{n}$ and $\psi^{n}$ at the time step $t^{n}$. We introduce the following finite-dimensional spaces:

$$
\mathbb{H}_{h}=\left\{\zeta_{h} \in \mathcal{C}^{0}(\bar{\Lambda}): \zeta_{h \mid K} \in P_{1}(K), \forall K \in \mathcal{T}_{h}\right\}, \quad \mathbb{G}_{h}=\mathbb{N}_{h} \cap H_{0}(\operatorname{div}, \Lambda),
$$




$$
\mathbb{N}_{h}=\mathbb{H}_{h}^{d}, \quad \mathbb{V}_{h}\left(\xi_{0}\right)=\mathbb{H}_{h} \cap H_{0}^{1}(\Lambda)+\pi_{h}\left(\widetilde{\xi}_{0}\right),
$$

where $\pi_{h}$ represents a $P_{1}$ Lagrange interpolant on the boundary $\partial \Lambda$. Given $\xi_{0} \in H^{1 / 2}(\partial \Lambda)$, the harmonic relevant $\widetilde{\xi_{0}}$ in the space $H^{1}(\Lambda)$ satisfy the following problem

$$
\triangle \widetilde{\xi}_{0}=0 \text { in } \Lambda \quad \text { such that } \quad \widetilde{\xi_{0}}=\xi_{0} \text { on } \partial \Lambda .
$$

Discretization of equation (2.14). Let us consider $\varphi_{h}^{n} \in \mathbb{V}_{h}\left(\varphi_{\partial \Lambda}\right)$ and we proceed to compute $\mathbf{n}_{h}^{n} \in \mathbb{N}_{h}$. The level set gradient vector $\nabla \varphi_{h}^{n} \in\left(P_{0}\right)^{d}, \forall K \in \mathcal{T}_{h}$. Let us denote by $\boldsymbol{g}_{h}^{n}$ by $L^{2}$ projection of $\nabla \varphi_{h}^{n}$ in $\mathbb{N}_{h}$, and we have

$$
\int_{\Lambda} \boldsymbol{g}_{h}^{n} \cdot \varsigma_{h}=\int_{\Lambda} \nabla \varphi_{h}^{n} \cdot \varsigma_{h}, \quad \forall \varsigma_{h} \in \mathbb{N}_{h}
$$

To assemble the left hand side of the equation (3.7), we use the lumped mass matrix, and the numerical computation of $\boldsymbol{g}_{h}^{n}$ is straightforward. Accordingly, the numerical approximation of the unit normal vector is given by

$$
\mathbf{n}_{h}^{n}\left(x_{i}\right)= \begin{cases}\frac{\boldsymbol{g}_{h}^{n}\left(x_{i}\right)}{\left|\boldsymbol{g}_{h}^{n}\left(x_{i}\right)\right|} & \text { if }\left|\boldsymbol{g}_{h}^{n}\left(x_{i}\right)\right| \neq 0 \\ \mathbf{0} & \text { otherwise }\end{cases}
$$

Discretization of equation (2.15). We consider an explicit scheme for the numerical approximation of $\gamma^{n}$. Given the discretized level set function $\varphi_{h}^{n} \in \mathbb{V}_{h}\left(\varphi_{\partial \Lambda}\right)$, the problem reads

find $\gamma_{h}^{n} \in \mathbb{G}_{h}$ such that for all $\tau \in \mathbb{G}_{h}$, we have

$$
\int_{\Lambda} \gamma_{h}^{n} \cdot \boldsymbol{\tau}+\int_{\Lambda}\left|\nabla \varphi_{h}^{n}\right| \nabla \cdot \tau=0
$$

Finally, with an exact evaluation of the multi-linear forms, the discrete problem $\mathcal{P}_{t}(3.1 \mathrm{a} / 3.1 \mathrm{~b})$ reads

$\mathcal{P}_{t, h}^{\star}$ : given $\varphi_{h}^{n} \in \mathbb{V}_{h}\left(\varphi_{0}\right), \psi_{h}^{n} \in \mathbb{H}_{h}, \mathbf{n}_{h}^{n} \in \mathbb{N}_{h}$ and $\gamma_{h}^{n} \in \mathbb{G}_{h}$,

find $\left(\varphi_{h}^{n+1}, \psi_{h}^{n+1}\right) \in \mathbb{V}_{h}\left(\varphi_{0}\right) \times \mathbb{H}_{h}$, such that $\forall(\zeta, \xi) \in \mathbb{V}_{h}(0) \times \mathbb{H}_{h}$, we have

$$
\begin{aligned}
& m\left(\left|\nabla \varphi_{h}^{n}\right|, \psi_{h}^{n+1}, \xi\right)-a\left(\left|\nabla \varphi_{h}^{n}\right| \mathbf{I}, \varphi_{h}^{n+1}, \xi\right)=2 m\left(\nabla \varphi_{h}^{n} \cdot \gamma_{h}^{n}, 1, \xi\right), \\
& a\left(\left|\nabla \varphi_{h}^{n}\right|^{3}\left(\mathbf{I}-\mathbf{n}_{h}^{n} \otimes \mathbf{n}_{h}^{n}\right), \psi_{h}^{n+1}, \zeta\right)+m\left(\frac{3\left|\nabla \varphi_{h}^{n}\right|^{3}}{2 \Delta t}, \varphi_{h}^{n+1}, \zeta\right) \\
& +a\left(\frac{\left|\nabla \varphi_{h}^{n}\right|}{2}\left(\psi_{h}^{n}\right)^{2} \mathbf{I}, \varphi_{h}^{n+1}, \zeta\right)=m\left(\frac{\left|\nabla \varphi_{h}^{n}\right|^{3}}{2 \Delta t}\left(4 \varphi_{h}^{n}-\varphi_{h}^{n-1}\right), 1, \zeta\right) \\
& -m\left(4\left|\nabla \varphi_{h}^{n}\right|^{2}\left(\mathbf{I}-\mathbf{n}_{h}^{n} \otimes \mathbf{n}_{h}^{n}\right) \nabla \psi_{h}^{n} \cdot \gamma_{h}^{n}+2\left(\psi_{h}^{n}\right)^{2}\left(\nabla \varphi_{h}^{n} \cdot \gamma_{h}^{n}\right)-p^{n}\left|\nabla \varphi_{h}^{n}\right|^{4}+\lambda^{n} \psi_{h}^{n}\left|\nabla \varphi_{h}^{n}\right|^{3}, 1, \zeta\right) .
\end{aligned}
$$

Using the finite element environment Rheolef [64], we can not perform the exact evaluation of the multi-linear forms in $\mathcal{P}_{t, h}^{\star}$. However, an approximation is introduced and the $P_{1}$ Lagrange interpolant $\pi_{h}$ is used to approximate the weights in the previous forms. Hence, we solve the following approximated problem

$\mathcal{P}_{t, h}$ : given $\varphi_{h}^{n} \in \mathbb{V}_{h}\left(\varphi_{0}\right), \psi_{h}^{n} \in \mathbb{H}_{h}, \mathbf{n}_{h}^{n} \in \mathbb{N}_{h}$ and $\gamma_{h}^{n} \in \mathbb{G}_{h}$,

find $\left(\varphi_{h}^{n+1}, \psi_{h}^{n+1}\right) \in \mathbb{V}_{h}\left(\varphi_{0}\right) \times \mathbb{H}_{h}$, such that $\forall(\zeta, \xi) \in \mathbb{V}_{h}(0) \times \mathbb{H}_{h}$, we have

$$
\begin{aligned}
& m\left(\pi_{h}\left[\left|\nabla \varphi_{h}^{n}\right|\right], \psi_{h}^{n+1}, \xi\right)-a\left(\pi_{h}\left[\left|\nabla \varphi_{h}^{n}\right| \mathbf{I}\right], \varphi_{h}^{n+1}, \xi\right)=2 m\left(\pi_{h}\left[\nabla \varphi_{h}^{n} \cdot \gamma_{h}^{n}\right], 1, \xi\right), \\
& a\left(\pi_{h}\left[\left|\nabla \varphi_{h}^{n}\right|^{3}\left(\mathbf{I}-\mathbf{n}_{h}^{n} \otimes \mathbf{n}_{h}^{n}\right)\right], \psi_{h}^{n+1}, \zeta\right)+\frac{3}{2 \triangle t} m\left(\pi_{h}\left[\left|\nabla \varphi_{h}^{n}\right|^{3}\right], \varphi_{h}^{n+1}, \zeta\right)+ \\
& \frac{1}{2} a\left(\pi_{h}\left[\left|\nabla \varphi_{h}^{n}\right|\left(\psi_{h}^{n}\right)^{2} \mathbf{I}\right], \varphi_{h}^{n+1}, \zeta\right)=\frac{1}{2 \triangle t} m\left(\pi_{h}\left[\left|\nabla \varphi_{h}^{n}\right|^{3}\left(4 \varphi_{h}^{n}-\varphi_{h}^{n-1}\right)\right], 1, \zeta\right)- \\
& m\left(\pi_{h}\left[4\left|\nabla \varphi_{h}^{n}\right|^{2}\left(\mathbf{I}-\mathbf{n}_{h}^{n} \otimes \mathbf{n}_{h}^{n}\right) \nabla \psi_{h}^{n} \cdot \gamma_{h}^{n}+2\left(\psi_{h}^{n}\right)^{2}\left(\nabla \varphi_{h}^{n} \cdot \gamma_{h}^{n}\right)-p^{n}\left|\nabla \varphi_{h}^{n}\right|^{4}+\lambda^{n} \psi_{h}^{n}\left|\nabla \varphi_{h}^{n}\right|^{3}\right], 1, \zeta\right) .
\end{aligned}
$$


Numerical computation of the residuals. The previous finite-dimensional linear system $\mathcal{P}_{t, h}$ involves the following matrix structure:

$$
\left(\begin{array}{cc}
A_{h}^{n} & B_{h}^{n} \\
C_{h}^{n} & D_{h}^{n}
\end{array}\right) \cdot\left(\begin{array}{c}
\Psi_{h}^{n+1} \\
\Phi_{h}^{n+1}
\end{array}\right)=\left(\begin{array}{c}
F_{h}^{n} \\
G_{h}^{n}
\end{array}\right)
$$

In the present work, this system is solved efficiently using the LU factorization of matrices performed by the UMFPACK library 1 within the $\mathrm{C}++$ finite element environment Rheole 264 . Since the weighted bilinear forms depend on $\left(\Psi_{h}^{n}, \Phi_{h}^{n}\right)^{T}$ at the previous time step, the linear system is assembled at each time step. It is required to have an appropriate convergence test for problems having a stationary solution. This test can possibly be based on the computation of the relative error. Nevertheless, this is not enough to assert that the solution converges and we decide that the convergence criterion is based on the computation of the residual of the problem $\mathcal{P}_{t, h}$ (3.10) 3.11 . Let us first consider the equation (3.1b) that includes the time derivative term. We denote by res ${ }_{I, h}^{n}$ the residual evaluated with the discrete $\mathrm{H}^{-1}$ norm at time $t^{n}$. For all $\zeta_{h} \in \mathbb{V}_{h}(0)$, the residual is given by

$$
\operatorname{res}_{I, h}^{n} \cdot \zeta_{h}^{T}=\left(\mathcal{A}_{h}^{n} \psi_{h}^{n}+\mathcal{B}_{h}^{n} \varphi_{h}^{n}-\mathcal{F}_{h}^{n}\right) \cdot \zeta_{h}^{T},
$$

where $\mathcal{A}_{h}^{n}, \mathcal{B}_{h}^{n}$ and $\mathcal{F}_{h}^{n}$ are the discrete operators induced by the forms contained in the equation (3.11). If the problem admits a stationary solution, as the problem of the equilibrium of RBCs, the residual verifies $\lim _{n \rightarrow \infty} \operatorname{res}_{I, h}^{n}=0$. For all $\zeta_{h} \in \mathbb{V}_{h}(0)$, we proceed with the computation of the discrete norm of the residual, denoted simply $|\cdot|_{\mathrm{H}_{\mathrm{h}}^{-1}}$. This norm is defined by duality and we have

$$
\begin{aligned}
\left|\operatorname{res}_{I, h}^{n}\right|_{\mathrm{H}_{\mathrm{h}}^{-1}} & \left.=\sup _{\zeta_{h} \in \mathbb{V}_{h}(0)} \frac{\left\langle\zeta_{h}, \operatorname{res}_{I, h}^{n}>_{\mathbb{V}_{h}(0), \mathrm{H}_{\mathrm{h}}^{-1}}\right.}{\left|v_{h}\right| \mathbb{V}_{h}(0)}=\sup _{\substack{v_{h} \in \mathbb{V}_{h}(0): \\
\left|v_{h}\right| \mathbb{V}_{h}(0)=1}}<v_{h}, \operatorname{res}_{I, h}^{n}\right\rangle_{\mathbb{V}_{h}(0), \mathrm{H}_{\mathrm{h}}^{-1}} \\
= & \max _{i \leq \operatorname{dim}\left(\mathbb{V}_{h}(0)\right)}<\overline{\phi_{i, h}}, \operatorname{res}_{I, h}^{n}>_{\mathbb{V}_{h}(0), \mathrm{H}_{\mathrm{h}}^{-1}}=\max _{x \in \# \operatorname{dof}\left(\mathbb{V}_{h}(0)\right)}\left|\operatorname{res}_{I, h}^{n}(x)\right|,
\end{aligned}
$$

where $\overline{\phi_{j, h}}$ represents the normalized nodal finite element basis of $\mathbb{V}_{h}(0)$. The integer $\operatorname{dim}\left(\mathbb{V}_{h}(0)\right)$ represents the dimension of $\mathbb{V}_{h}(0)$, and \#dof $\left(\mathbb{V}_{h}(0)\right)$ denotes the set of nodes associated to the degrees of freedom of $\mathbb{V}_{h}(0)$. Remark that, while the elements of $\mathbb{V}_{h}(0)$ vanishes on the boundary, the set \# dof $\left(\mathbb{V}_{h}(0)\right)$ contains all the nodes associated to the degrees of freedom of $\mathbb{V}_{h}(0)$ with the exception of those located on the boundary. Finally, we notice that we approximate the computation of the residual $\operatorname{res}_{I I, h}^{n}$ of the equation (3.10), and we evaluate it in a similar way as $\operatorname{res}_{I, h}^{n}$.
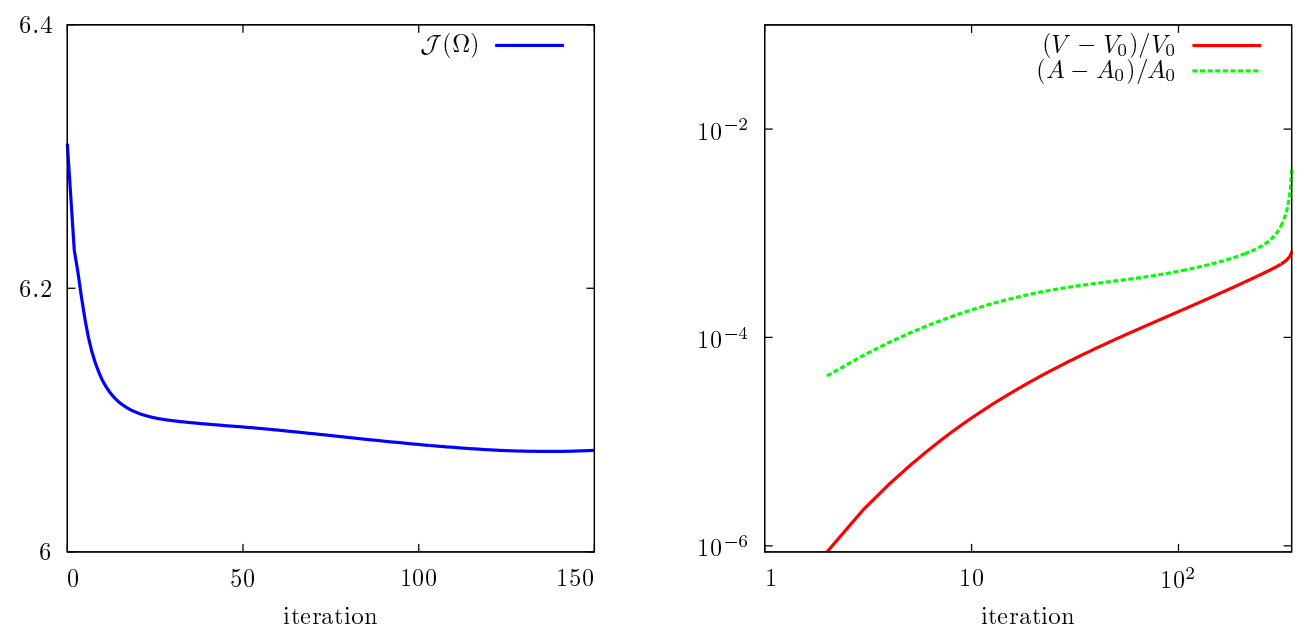

Figure 5: Preservation of the volume and the area. (left) Evolution of the energy of Canham and Helfrich. (right) Plot in the logarithmic scale of the relative errors with respect to the iterations.

${ }^{1}$ UMFPACK routines - http://www.cise.ufl.edu/research/sparse/umfpack/

${ }^{2}$ Rheolef - http://www-ljk.imag.fr/membres/Pierre.Saramito/rheolef/ 


\subsection{Numerical verification of the volume and area constraints}

We consider a cell having reduced area $\gamma=0.75$ and we solve the problem $\mathcal{P}_{t, h}$ (3.11) following Algorithm 2 Let us denote by $V_{0}$ and $A_{0}$ the surface area and the perimeter of the cell at the initial time $t=0$. We plot in the figure 5 the evolution of the energy of Canham and Helfrich and the relative error in area and perimeter. Although we observe that the energy $\mathcal{J}$ is decreasing, results show that the constraints of area and perimeter are not verified and an error is accumulated significantly over the iterations. However, the errors made in the preservation of the constraints were expected. Indeed, regarding the preservation of the surface, the constraint $\int_{\Omega} 1=V_{0}$ was replaced by the equation $\frac{d}{d t} \int_{\Omega} 1=0$ to allow the computation of the Lagrange multipliers. Notice that the two equations does not describe the same constraint but they should be equivalent up to a constant value. This value could represent therefore the numerical error coming, for example, from the rounding and the interpolations between spaces. The same argument is also valid for the constraint of the volume preservation. Thereafter, the idea is to restore the area and the perimeter lost by adding an appropriate correction prior to the calculation of the optimal Lagrange multipliers $p^{*}$ and $\lambda^{*}$. These analytical computations are performed in the formal manner, and we assume to have enough regularity in the integral calculus.

$\boldsymbol{A}$ posteriori correction of the Lagrange multipliers. To overcome the problems of mass preservation, we introduce two parameters $\varepsilon_{V}^{n}$ and $\varepsilon_{A}^{n}$ needed for the correction of errors in the equations 3.3 and 3.4, respectively. These parameters vary in time, and they are given at the time $t^{n}$ by

$$
\varepsilon_{A}^{n}=\frac{A_{0}-\int_{\Gamma^{n}} 1 \mathrm{ds}}{\Delta t} \quad \text { and } \quad \varepsilon_{V}^{n}=\frac{\int_{\Omega^{n}} 1 \mathrm{dx}-V_{0}}{\Delta t} .
$$

The corrections are applied and the problem (2.8a) is replaced by

$$
\begin{aligned}
& \frac{\partial \varphi}{\partial t}+\left[\Delta_{s} H-H \nabla \cdot\left(\frac{\nabla_{s}|\nabla \varphi|}{|\nabla \varphi|}\right)-\frac{1}{2} \nabla \cdot\left(H^{2} \mathbf{n}\right)-p-\lambda H\right]|\nabla \varphi|=0 \quad \text { in }(0, T) \times \Lambda, \\
& \frac{d}{d t} \int_{\Lambda} \mathscr{H}(\varphi)=\varepsilon_{V}^{n} \quad \text { in }(0, T), \\
& \frac{d}{d t} \int_{\Gamma} 1=\varepsilon_{A}^{n} \quad \text { in }(0, T) .
\end{aligned}
$$

By using (3.14) and the Reynolds formulae, we obtain

$$
\frac{d}{d t} \int_{\Gamma} 1=\varepsilon_{A}^{n} \Leftrightarrow p \int_{\Gamma} H+\lambda \int_{\Gamma} H^{2}+\varepsilon_{A}^{n}=\int_{\Gamma} H \Delta_{s} H+\int_{\Gamma} H^{2} \nabla \cdot\left(\frac{\nabla_{s}|\nabla \varphi|}{|\nabla \varphi|}\right)-\frac{1}{2} \int_{\Gamma} H \nabla \cdot\left(H^{2} \mathbf{n}\right) .
$$

Using the equation (3.13), we have

$$
\frac{d}{d t} \int_{\Lambda} \mathscr{H}(\varphi)=\varepsilon_{V}^{n} \Leftrightarrow \int_{\Lambda} \frac{\partial \varphi}{\partial t} \delta(\varphi)=\varepsilon_{V}^{n} \Leftrightarrow p \int_{\Gamma} 1+\lambda \int_{\Gamma} H-\varepsilon_{V}^{n}=\int_{\Gamma} \Delta_{s} H+\int_{\Gamma} H \nabla \cdot\left(\frac{\nabla_{s}|\nabla \varphi|}{|\nabla \varphi|}\right)-\frac{1}{2} \int_{\Gamma} \nabla \cdot\left(H^{2} \mathbf{n}\right) .
$$

Collecting the element above, the corrected Lagrange multipliers are given by

$\lambda=\frac{\int_{\Gamma} 1 \times\left(-\varepsilon_{A}^{n}+\int_{\Gamma} H^{2} \nabla \cdot\left(\frac{\nabla_{s}|\nabla \varphi|}{|\nabla \varphi|}\right)-\left|\nabla_{s} H\right|^{2}-\frac{H}{2} \nabla \cdot\left(H^{2} \mathbf{n}\right)\right)-\int_{\Gamma} H \times\left(\varepsilon_{V}^{n}+\int_{\Gamma} H \nabla \cdot\left(\frac{\nabla_{s}|\nabla \varphi|}{|\nabla \varphi|}\right)-\frac{1}{2} \nabla \cdot\left(H^{2} \mathbf{n}\right)\right)}{\int_{\Gamma} 1 \int_{\Gamma} H^{2}-\left(\int_{\Gamma} H\right)^{2}}$

$p=\frac{\varepsilon_{V}^{n} \int_{\Gamma} H \nabla \cdot\left[\frac{\nabla_{s}|\nabla \varphi|}{|\nabla \varphi|}\right]-\frac{1}{2} \int_{\Gamma} \nabla \cdot\left(H^{2} \mathbf{n}\right)-\lambda \int_{\Gamma} H}{\int_{\Gamma} 1}$ 
Remark that the corrections of the Lagrange multipiers can be interpreted as a posteriori control on the values of $\lambda^{*}$ (3.5) and $p^{*}$ (3.6). Thus, we have $\lambda=\lambda^{*}+\delta \lambda^{n}, p=p^{*}+\delta p^{n}$, where

$$
\delta \lambda^{n}=\frac{-\varepsilon_{A}^{n} \int_{\Gamma} 1-\varepsilon_{V}^{n} \int_{\Gamma} H}{\int_{\Gamma} 1 \int_{\Gamma} H^{2}-\left(\int_{\Gamma} H\right)^{2}} \quad \text { and } \quad \delta p^{n}=\frac{\varepsilon_{A}^{n} \int_{\Gamma} 1 \int_{\Gamma} H+\varepsilon_{V}^{n} \int_{\Gamma} 1 \int_{\Gamma} H^{2}}{\int_{\Gamma} 1\left(\int_{\Gamma} 1 \int_{\Gamma} H^{2}-\left(\int_{\Gamma} H\right)^{2}\right)} .
$$

Observe that the corrections a posteriori of the Lagrange multipliers $\delta \lambda^{n}$ and $\delta p^{n}$ (3.17) vanish if the parameters $\varepsilon_{V}^{n}$ and $\varepsilon_{A}^{n}$ are null. The modified algorithm used to solve the saddle point problem is detailed in Algorithm 3 , and we illustrate a full description of the numerical strategy in the graph 6 .
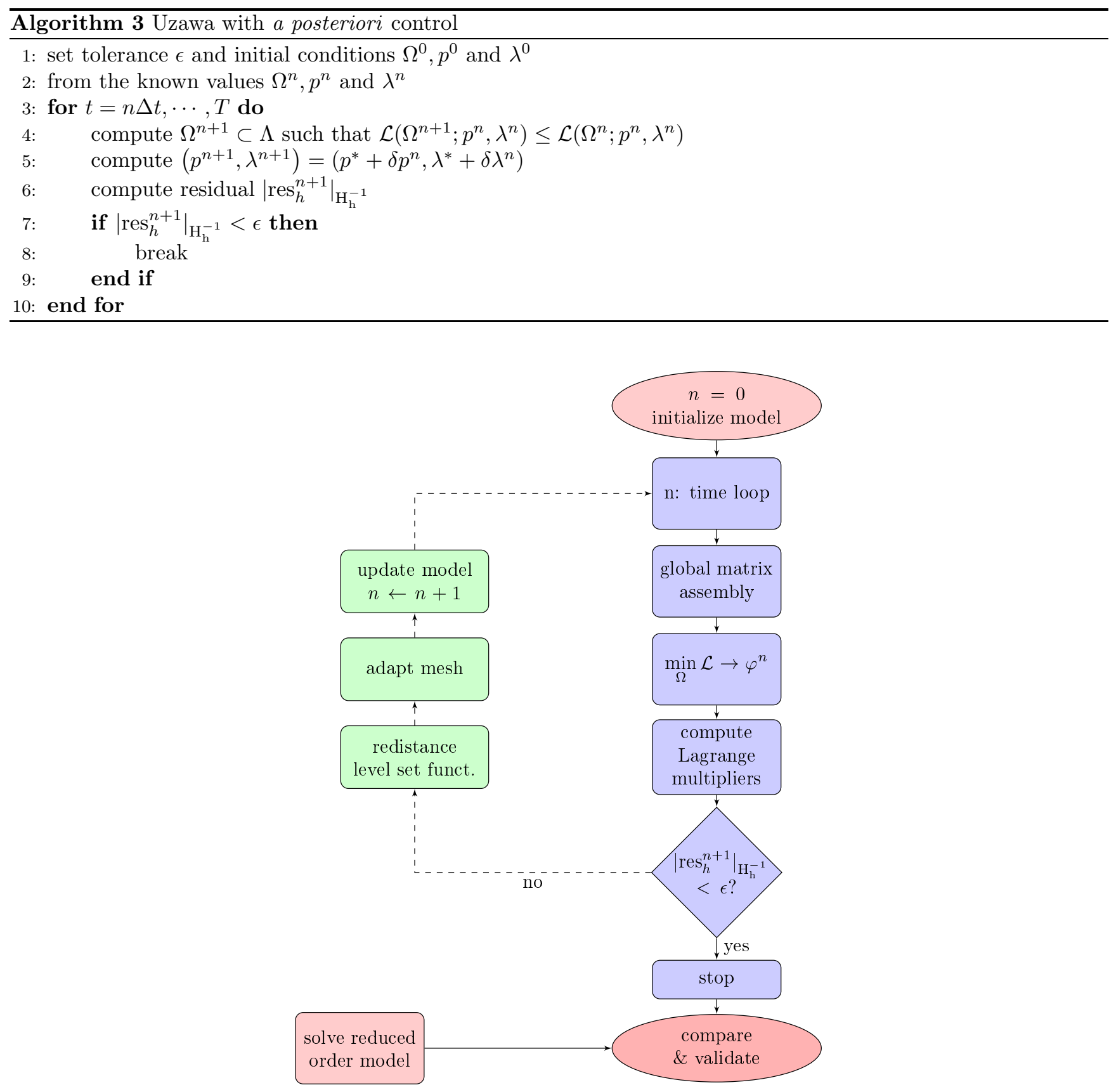

Figure 6: A Graphical illustration of the numerical method. 


\section{Reduced order model in the two-dimensional case}

Notations. Let us consider a functional $f$ that depends on a function $\varphi(x)$, where $x$ represents the spatial coordinate. We introduce the following notations

$$
f^{\prime}(x):=\frac{d f}{d x}(x) \quad \text { and } \quad f_{\psi}^{\prime}(\varphi):=\frac{d f}{d \varphi}(\varphi)(\psi)
$$

In this section, we write a reduced order model that describes the equilibrium state of the cell as the solution of an ordinary differential equation (ODE). We assume, as an approximation, that the cell is symmetric with respect to the horizontal axis (i.e. x-axis) and the vertical axis (i.e. z-axis). The solution of this reduced order model will help afterwards to validate the solution of the finite element solver, as depicted in the graph 6 .

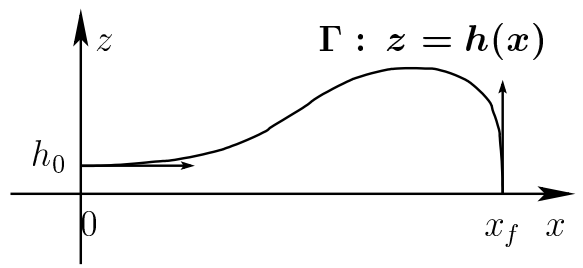

Figure 7: Cartesian notations used in the two-dimensional case

Mathematical formulation. In the equilibrium state, the membrane is described using a parametric representation $z=h(x)$, see Fig. 17. We denote by $x_{f}$ the maximum radius of the membrane, and we have $h\left(x_{f}\right)=0$. The membrane is described by the parametric representation

$$
\Gamma=\left\{\mathbf{X}(x, z)=(x, z(x)):(x, z) \in\left[0, x_{f}\right] \times[0,+\infty)\right\}
$$

We introduce the function $\phi(x, z)=z-h(x)$. The membrane is then described as a level set function $\Gamma=$ $\left\{x \in\left[0, x_{f}\right] \times[0,+\infty]: \phi(x, z)=0\right\}$. An infinetisimal length on $\Gamma$ writes $\mathrm{dl}=\left(1+\left(h^{\prime}(x)\right)^{2}\right)^{\frac{1}{2}} \mathrm{~d} x$, while the area $V_{0}$ and the perimeter $A_{0}$ write

$$
V_{0}=4 \int_{0}^{x_{f}} h(x) \mathrm{d} x=-4 \int_{0}^{x_{f}} w(x) x \mathrm{~d} x \quad \text { and } \quad A_{0}=4 \int_{0}^{x_{f}}\left(1+w^{2}(x)\right)^{\frac{1}{2}} \mathrm{~d} x,
$$

where $w(x)=h^{\prime}(x)$. In the following, we write the problem with respect to $w$. The outward unit normal vector $\mathbf{n}$ and the mean curvature $H$ are given respectively by

$$
\mathbf{n}(x)=\frac{\nabla \phi}{|\nabla \phi|}=\frac{1}{\left(1+w(x)^{2}\right)^{\frac{1}{2}}}\left(\begin{array}{c}
-w(x) \\
1
\end{array}\right) \quad \text { and } \quad H(x, z)=\nabla \cdot \mathbf{n}=-\frac{w^{\prime}(x)}{\left(1+w(x)^{2}\right)^{\frac{3}{2}}} .
$$

The Lagrangian functional writes

$$
\frac{\mathcal{L}(\Omega ; \lambda, p)}{2}=\int_{0}^{x_{f}}\left[\left(H-H_{0}\right)^{2}+\lambda\right]\left(1+w^{2}(x)\right)^{\frac{1}{2}} \mathrm{~d} x-2 p \int_{0}^{x_{f}} x w(x) \mathrm{d} x .
$$

To write the Euler-Lagrange equation, we look for for the saddle-points of $\mathcal{L}(4.3$. Let us assume that $\psi(x)$ represents a regular function with a compact support. Therefore, we compute the Fréchet derivatives with respect to $w$ in the direction $\psi$. We have

$$
\begin{gathered}
{\left[\left(H-H_{0}\right)^{2}\right]_{\psi}(w)=2 H_{\psi}(w)\left(H-H_{0}\right) \quad \text { and } \quad\left[\left(1+w^{2}(x)\right)^{\frac{1}{2}}\right]_{\psi}(w)=\frac{w(x) \psi}{\left(1+w^{2}(x)\right)^{\frac{1}{2}}}} \\
\frac{\mathcal{L}^{\prime}(w ; \lambda, p)_{\psi}(w)}{2}=2 \int_{0}^{x_{f}} H_{\psi}(w)\left(H-H_{0}\right)\left(1+w^{2}(x)\right)^{\frac{1}{2}}+\int_{0}^{x_{f}} \frac{w \psi\left[\left(H-H_{0}\right)^{2}+\lambda\right]}{\left(1+w^{2}(x)\right)^{\frac{1}{2}}}-2 p \int_{0}^{x_{f}} x \psi
\end{gathered}
$$


From the expression of $\mathrm{H} 4.3$, we get

$$
H^{\prime}(x)=-\frac{\left[\left(1+w(x)^{2}\right) w^{\prime \prime}(x)-3 w(x) w^{\prime 2}(x)\right]}{\left(1+w(x)^{2}\right)^{\frac{5}{2}}} \quad \text { and } \quad H_{\psi}^{\prime}(w)=-\frac{\psi^{\prime}\left(1+w(x)^{2}\right)-3 w(x) w^{\prime}(x) \psi}{\left(1+w(x)^{2}\right)^{\frac{5}{2}}} .
$$

We denote respectively by $T_{1}, T_{2}$ and $T_{3}$ the three terms in the derivative of the Lagrangian, see Eq. (4.4).

$$
\begin{aligned}
T_{1} & =-\int_{0}^{x_{f}} \frac{2\left(H-H_{0}\right)}{\left(1+w(x)^{2}\right)^{2}}\left[\left(1+w(x)^{2}\right) \psi^{\prime}-3 w(x) w^{\prime}(x) \psi\right] \mathrm{d} x \\
& =-\left[\frac{2\left(H-H_{0}\right)}{\left(1+w(x)^{2}\right)} \psi\right]_{0}^{x_{f}}+\int_{0}^{x_{f}} \frac{\partial}{\partial x}\left[\frac{2\left(H-H_{0}\right)}{\left(1+w(x)^{2}\right)}\right] \psi \mathrm{d} x+\int_{0}^{x_{f}} \frac{6 w(x) w^{\prime}(x)\left(H-H_{0}\right)}{\left(1+w(x)^{2}\right)^{2}} \psi \mathrm{d} x
\end{aligned}
$$

From $w\left(x_{f}\right)=-\infty$, we obtain

$$
\frac{\partial}{\partial x}\left[\frac{2\left(H(x)-H_{0}\right)}{\left(1+w(x)^{2}\right)}\right]=\frac{2 H^{\prime}(x)}{\left(1+w(x)^{2}\right)}-\frac{4\left(H(x)-H_{0}\right) w(x) w^{\prime}(x)}{\left(1+w(x)^{2}\right)^{2}} .
$$

We end with

$$
T_{1}=\int_{0}^{x_{f}} \frac{2 H^{\prime}(x)}{\left(1+w(x)^{2}\right)} \psi \mathrm{d} x+\int_{0}^{x_{f}} \frac{2 w(x) w^{\prime}(x)\left(H(x)-H_{0}\right)}{\left(1+w(x)^{2}\right)^{2}} \psi \mathrm{d} x .
$$

By using the expression of $H^{\prime}(x)$ (4.5), we evaluate $T_{1}$ (4.6)

$$
\frac{2 H^{\prime}(x)}{\left(1+w(x)^{2}\right)}+\frac{2 w(x) w^{\prime}(x)\left(H(x)-H_{0}\right)}{\left(1+w(x)^{2}\right)^{2}}=-\frac{2 w^{\prime \prime}(x)}{\left(1+w(x)^{2}\right)^{\frac{5}{2}}}+\frac{4 w(x) w^{\prime 2}(x)}{\left(1+w(x)^{2}\right)^{\frac{7}{2}}}-\frac{2 w(x) w^{\prime}(x) H_{0}}{\left(1+w(x)^{2}\right)^{2}} .
$$

We have $\left(H(x)-H_{0}\right)^{2}+\lambda=\frac{w^{\prime}(x)^{2}}{\left(1+w(x)^{2}\right)^{3}}+\frac{2 H_{0} w^{\prime}(x)}{\left(1+w(x)^{2}\right)^{\frac{3}{2}}}+\left(H_{0}^{2}+\lambda\right)$. We get

$$
T_{2}=\int_{0}^{x_{f}}\left[\frac{w(x) w^{\prime 2}(x)}{\left(1+w(x)^{2}\right)^{\frac{7}{2}}}+\frac{2 H_{0} w^{\prime}(x) w(x)}{\left(1+w(x)^{2}\right)^{2}}+\frac{\left(H_{0}^{2}+\lambda\right) w(x)}{\left(1+w(x)^{2}\right)^{\frac{1}{2}}}\right] \psi \mathrm{d} x .
$$

By using $T_{1}$ (4.6) and $T_{2}$ (4.7), the equation (4.4) reads

$$
\frac{\mathcal{L}^{\prime}(w ; \lambda, p)_{\psi}(w)}{2}=\int_{0}^{x_{f}}\left[-\frac{2 w^{\prime \prime}(x)}{\left(1+w(x)^{2}\right)^{\frac{5}{2}}}+\frac{5 w(x) w^{\prime 2}(x)}{\left(1+w(x)^{2}\right)^{\frac{7}{2}}}+\frac{\left(H_{0}^{2}+\lambda\right) w(x)}{\left(1+w(x)^{2}\right)^{\frac{1}{2}}}-2 p x\right] \psi \mathrm{d} x .
$$

Therefore, the Euler-Lagrange equation describing the RBC's equilibrium reads

$$
\frac{2 w^{\prime \prime}(x)}{\left(1+w(x)^{2}\right)^{\frac{5}{2}}}=\frac{5 w(x) w^{\prime 2}(x)}{\left(1+w(x)^{2}\right)^{\frac{7}{2}}}+\frac{\left(H_{0}^{2}+\lambda\right) w(x)}{\left(1+w(x)^{2}\right)^{\frac{1}{2}}}-2 p x .
$$

By considering the suitable initial data and boundary conditions, the reduced order problem writes

$$
\left\{\begin{aligned}
\frac{2 w^{\prime \prime}(x)}{\left(1+w(x)^{2}\right)^{\frac{5}{2}}} & =\frac{5 w(x) w^{\prime 2}(x)}{\left(1+w(x)^{2}\right)^{\frac{7}{2}}}+\frac{\left(H_{0}^{2}+\lambda\right) w(x)}{\left(1+w(x)^{2}\right)^{\frac{1}{2}}}-2 p x, \quad 0<x<x_{f} \\
w(0) & =0 \\
w^{\prime}(0) & =w_{0}^{\prime} .
\end{aligned}\right.
$$

We notice that the condition $w_{0}^{\prime}>0$ is imposed if we expect a biconcave shape. To decrease the derivation order in (4.9), we introduce a variable change $\kappa(x)=\frac{w(x)}{\left(1+w(x)^{2}\right)^{\frac{1}{2}}}$, and we write the problem (4.9) with respect to $\kappa(x)$. The boundary condition is $\kappa(0)=0$, and we have

$$
\kappa^{\prime}(x)=\frac{w^{\prime}(x)}{\left(1+w^{2}(x)\right)^{3 / 2}} \quad \text { and } \quad \kappa^{\prime \prime}(x)=\frac{w^{\prime \prime}(x)}{\left(1+w(x)^{2}\right)^{\frac{3}{2}}}-\frac{3 w(x) w^{\prime 2}(x)}{\left(1+w(x)^{2}\right)^{\frac{5}{2}}} .
$$


Remark that $\kappa^{\prime}(0)=w^{\prime}(0)$. The two variables $\kappa(x)$ and $w(x)$ have the same sign, and we have $w(x)=\frac{\kappa(x)}{\left(1-\kappa^{2}(x)\right)^{\frac{1}{2}}}$.

The mean curvature is given by $H(x)=-\kappa^{\prime}(x)$. We introduce the polynomial $P(\xi)=\left(H_{0}^{2}+\lambda\right) \xi-2 p x$. The reduced order problem writes

$$
\left\{\begin{aligned}
\kappa^{\prime \prime} & =\frac{P(\kappa)(x)-\kappa(x) \kappa^{2}(x)}{2\left(1-\kappa^{2}\right)}, 0<x<x_{f} \\
\kappa(0) & =0 \\
\kappa^{\prime}(0) & =w^{\prime}(0) .
\end{aligned}\right.
$$

Numerical discretization. To solve the problem (4.10), the derivation order is first reduced by introducing the variables $y(x)=\left(y_{1}, y_{2}\right)=\left(\kappa(x), \kappa^{\prime}(x)\right), y_{0}=\left(0, w^{\prime}(0)\right)$ and $f(y)=\left(y_{2}, \frac{P\left(y_{1}\right)-y_{1} y_{2}^{2}}{2\left(1-y_{1}^{2}\right)}\right)^{T}$. A first-order ordinary differential equation depending on $y$ is obtained

$$
y^{\prime}=f(y), \quad \text { if } 0<r<x_{f} \quad \text { and } \quad y(0)=y_{0} .
$$

Looking for biconcave shapes of RBCs, we solve the problem 4.11 in the intervalle [0, $\left.x_{f}\right]$, and we choose $x_{f}$ large enough such that $1-\kappa^{2}(x)$ changes the signum on a particular spatial position $x_{c}$ and it becomes negative in the intervalle $\left(x_{c}, x_{f}\right)$. A shooting method is used to obtain the position $x_{f}$. Finally, we compute

$$
w(x)=\frac{\kappa}{\left(1-\kappa^{2}\right)^{1 / 2}}, \quad 0<x<x_{f} \quad \text { and } \quad h(x)=\int_{x_{f}}^{x} w(x) \mathrm{d} x .
$$

\section{Numerical Simulations}
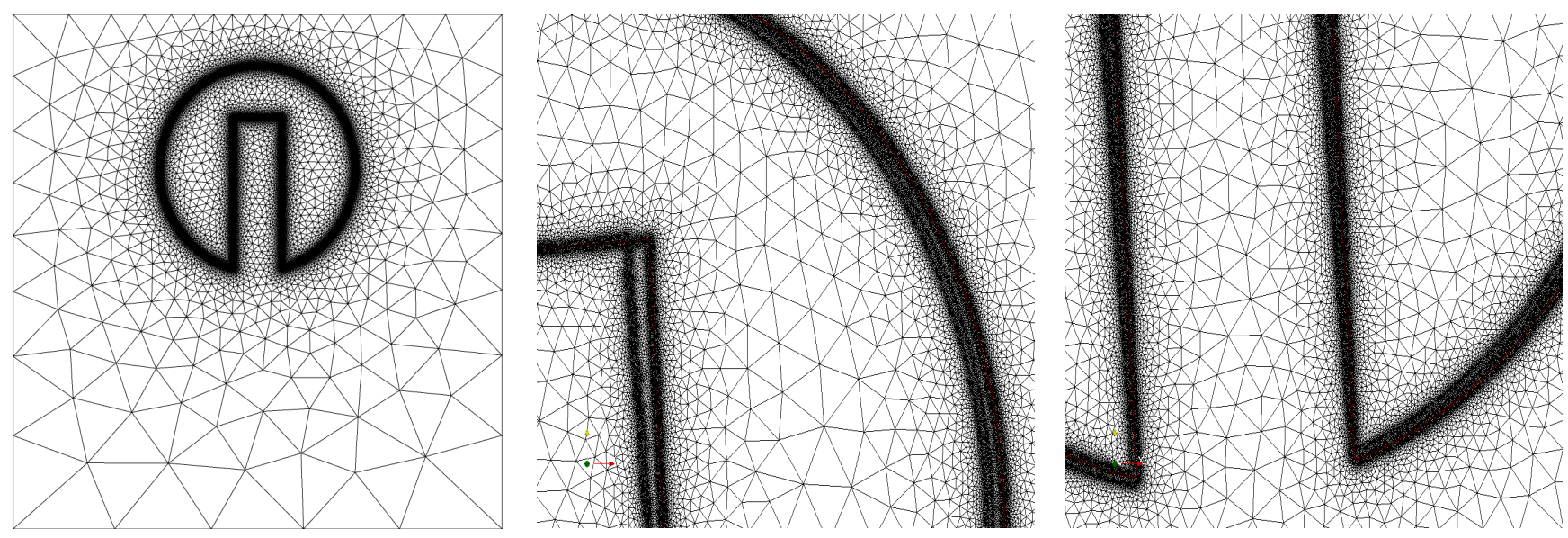

Figure 8: Example 1: Adapted mesh in the Zalesak's test.

\subsection{Software implementation}

The presented method has been implemented using the Rheolef environment3 [64], which is a general purpose $\mathrm{C}++$ library for scientific computing, with special emphasis on finite elements and parallel computation. Rheolef provides support for distributed-memory parallelism via MP 4 . Rheolef relies upon the Trilino: 5 , Boost 6 , Bla: 4 and

\footnotetext{
${ }^{3}$ Rheolef - http://www-ljk.imag.fr/membres/Pierre.Saramito/rheolef/

${ }^{4}$ Message passing interface - http://www.mpich.org

${ }^{5}$ Trilinos - http://trilinos.org

${ }^{6}$ Boost libraries - http://www.boost.org

${ }^{7}$ BLAS, Basic Linear Algebra Subprograms library - http://www.netlib.org/blas
} 
UMFPACK 8 libraries for much of its functionality. Rheolef bases on Scotch for distributed mesh partitioning 9 . The reduced order problem has been numerically solved with the free software package GNU Octave 24] 10 .

\subsection{Validation of the numerical solver}

We present in this section a set of numerical examples illustrating the main features and the accuracy of the numerical method. We focus, in the first part, on our mesh adapting tool.
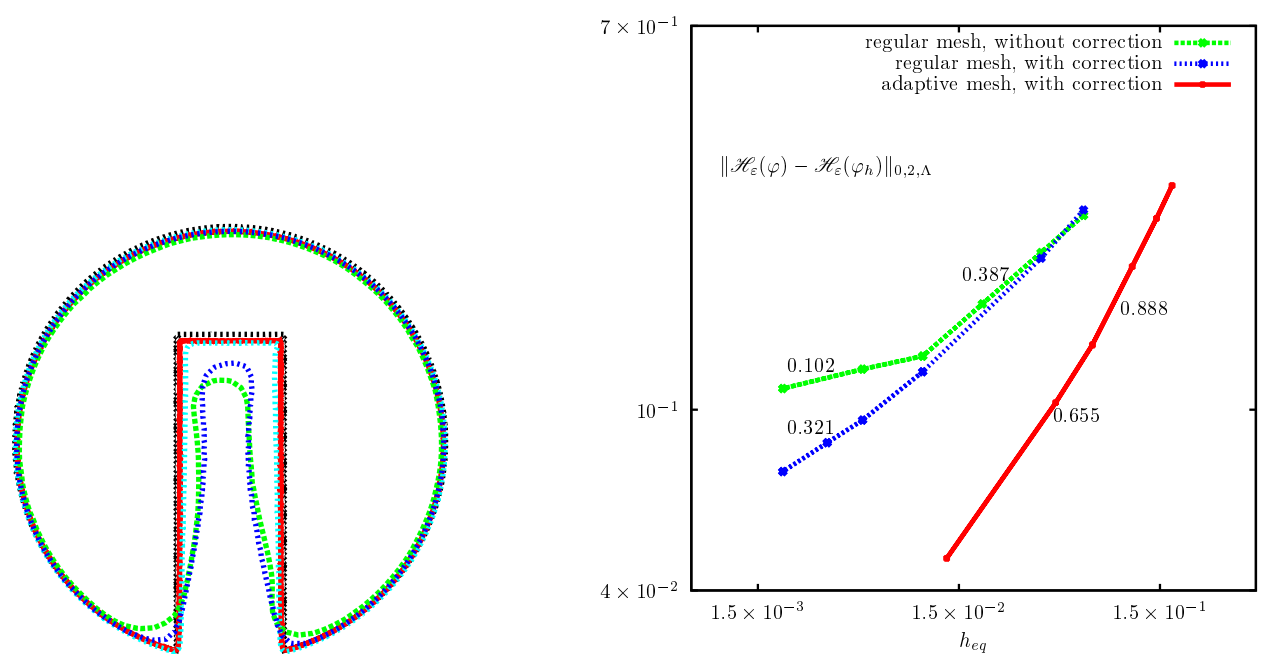

Figure 9: Example 1: (left) Contour plots of the rotating slotted circle after one rotating period: exact solution (black and dotted), regular mesh without mass correction: see Eq. 2.5 (green and dashed), regular mesh with mass correction (blue and dotted line), and adaptive mesh with mass correction (red and solid line). (right) Convergence properties in log-log scale.

\subsubsection{Exemple 1: The test of Zalesak's rigid disk}

We first study the motion of a rigid body under the effect of a prescribed rotational motion. This test has been initially proposed by Zalesak 75]. It has become one of the most common test for interface propagation used to test numerical methods, see e.g [76, 54, 74, 58, 78]. We are interested in the rotation of a slotted circle with a radius of $1 / 5$, the slot depth is $3 / 10$ and the width is equal to $1 / 10$. The slotted circle is initially centered at $(0.5,7 / 10)$ and we consider a rotational velocity field given by $\boldsymbol{u}=\left(\frac{\pi}{314}(50-y), \frac{\pi}{314} \cos (x-50)\right)^{T}$. The computational domain is the square $[0,1]^{2}$. The slotted circle completes one revolution after one period. Several mesh sizes were considered to check the mesh dependency and the convergence properties of our method. We plot in Fig. 9 the convergence of the error between the computed solution $\varphi_{h}$ and the exact solution $\varphi$ with respect to the equivalent space discretization $h_{e q}=1 / \sqrt{N_{n}}$, where $N_{n}$ represents the number of nodes in the adapted mesh, see Section 3.3. The error is plotted in the logarithmic scale and results show that we obtain better convergence slope firstly by adding the mass correction in the redistancing problem and secondly by using the mesh adaptation technique. Regarding the mesh adaptation, we consider the hessian matrix of the field $\Xi_{h}^{n}=\delta_{\varepsilon}\left(\varphi_{h}^{n}\right)+\delta_{\varepsilon}\left(\varphi_{h}^{n-1}\right)$. Fig 8 shows the accuracy of our meshing tool, in particular concerning the capture of the corners in the slotted disk.

\subsubsection{Exemple 2: The test of Leveque's deformable disk}

We study the advection of a deformable object in a shear flow field. This problem is used to test the ability of the numerical method to resolve and maintain thin filaments and to capture, afterwards, some particular biconcave shapes of red blood cells. This test was proposed by Leveque [50], and it is widely used to test a lot of numerical methods and strategies [26, 42, 63]. The domain of interest is $\Lambda=[0,1]^{2}$. We consider an initial deformable circle

\footnotetext{
${ }^{8}$ UMFPACK routines - http://www.cise.ufl.edu/research/sparse/umfpack/

${ }^{9}$ Scotch - http://www.labri.fr/perso/pelegrin/scotch

${ }^{10}$ Octave - www.gnu.org/software/octave/
} 

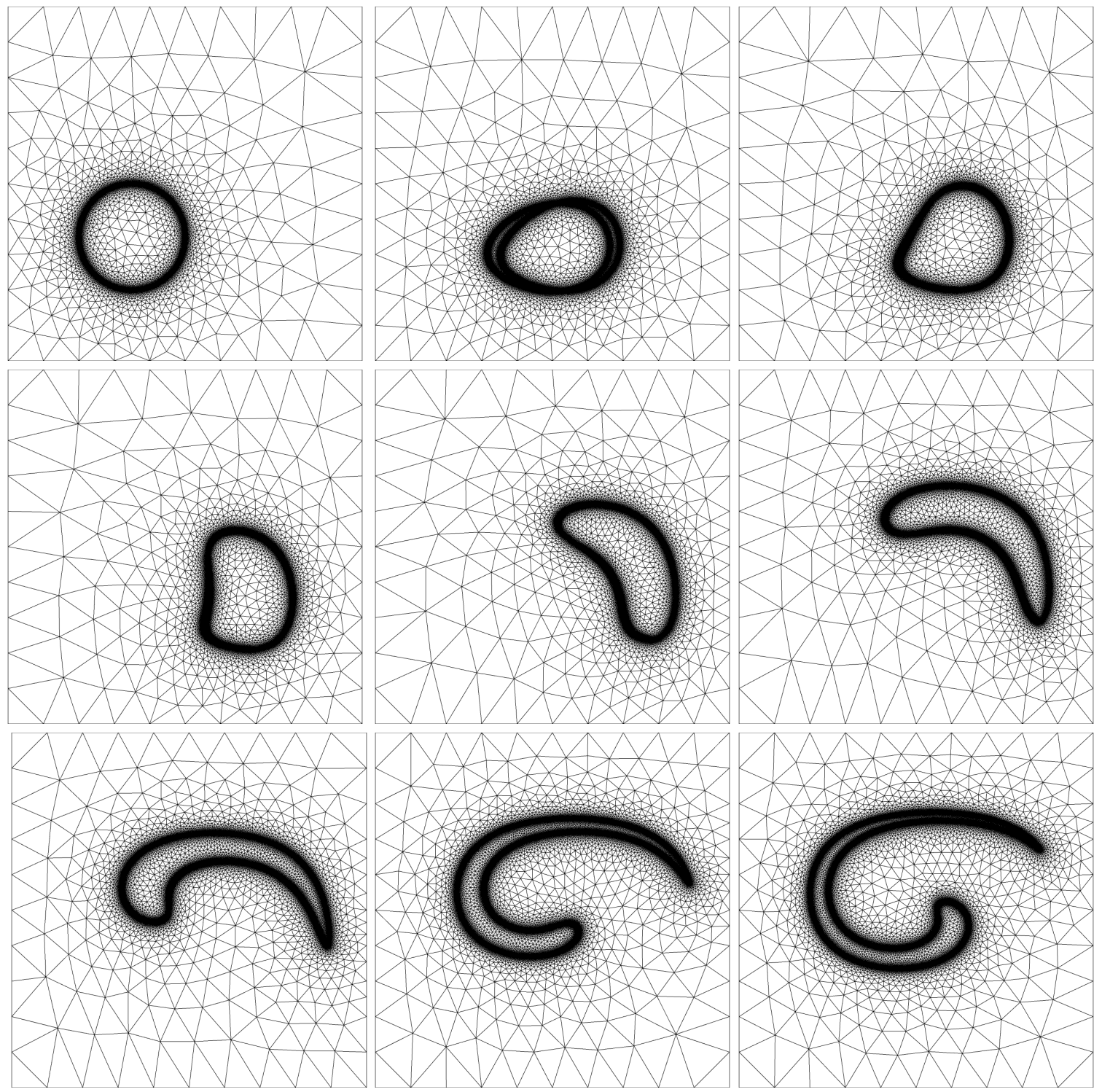

Figure 10: Example 2: Some adaptive meshes obtained in the test of Leveque's deformable circle.

with a radius 0.15 and centered on $(0.35,0.35)$. The circle is advected in a vortex with a periodic velocity field given by $\mathbf{u}(x, y)=\left(2(\sin (\pi x))^{2} \sin (2 \pi y) \sin (\pi t),-(\sin (\pi y))^{2} \sin (\pi t) \sin (2 \pi x)\right)^{T}$. It reaches the maximum of deformation at the middle of the time period. The velocity components change then their sign, and the deformable circle should reach its initial position at the end of the period. This test shows the ability of our mesh technique to detect the thin filaments, as depicted in Fig. 10.

\subsubsection{Exemple 3: Mean curvature motion}

We study the mean curvature motion. We are interested in the time evolution of $\Gamma_{0}=\partial \Omega_{0}$ such that, at each time $t \in(0, T)$, the curve $\Gamma(t)=\partial \Omega(t)$ moves with a normal speed equal to the mean curvature; the advection vector is given by $\boldsymbol{u}=-\mathrm{H} \boldsymbol{n}$. This problem is equivalent to the following shape optimization problem:

$$
\Omega=\underset{w}{\arg \min } \int_{\partial w} 1 \mathrm{ds} .
$$

The behavior of the evolution of a circle by mean curvature is well-known. The initial cercle remains a circle and shrinks into a point in a finite time. We solve this problem to test the mesh adaptation technique for a problem 

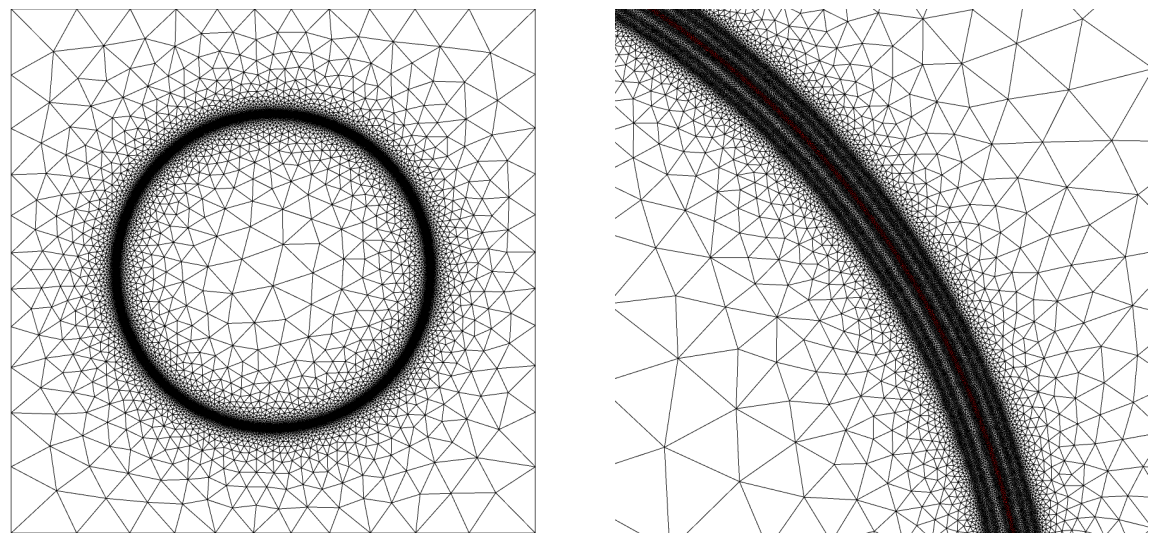

Figure 11: Example 3: (left) Adaptive mesh. (right): Zoom showing the effect of using the adaptive criterion.
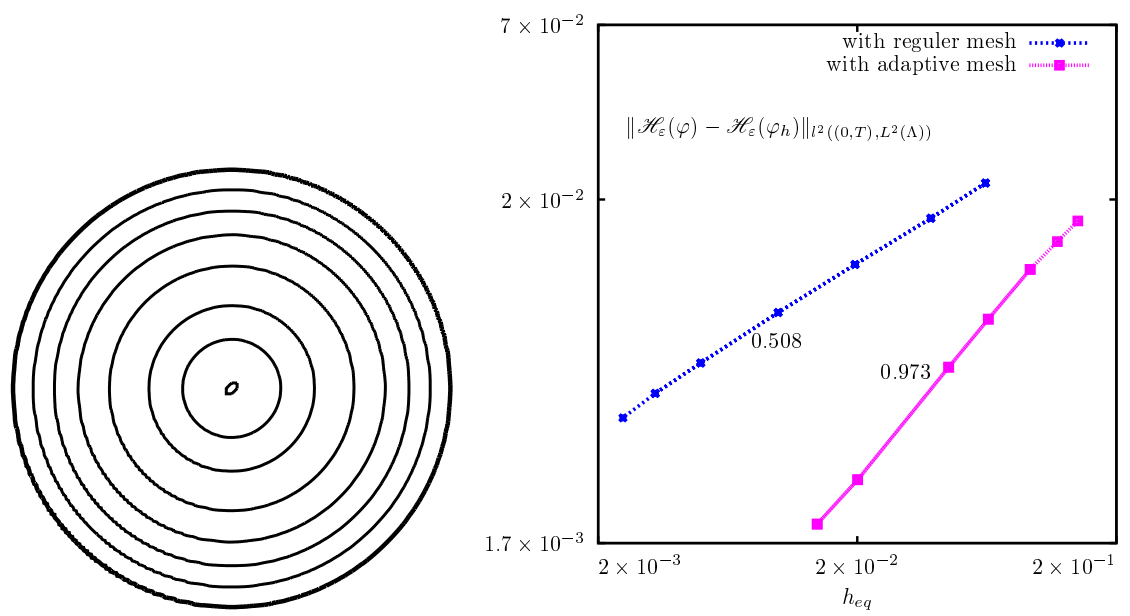

Figure 12: Example 3: (left) Evolution of a circle that shrinks into a point in a finite time. (right) Convergence properties in the $l^{2}\left((0, T) ; L^{2}(\Lambda)\right)$ norm. The logarithmic scale is used.

that evolves also the mean curvature. The mean curvature is an important quantity in the modelization of RBCs and we have to compute this quantity in an accurate way. To adapt the mesh, we choose the metric as the hessian of the field $\Xi_{h}^{n}=\delta_{\varepsilon}\left(\varphi_{h}^{n}\right)+\delta_{\varepsilon}\left(\varphi_{h}^{n+0.5}\right)+\delta_{\varepsilon}\left(\varphi_{h}^{n-1}\right)$ where $\varphi_{h}^{n+0.5}$ represents a first approximation of the solution at $t^{n+1}$, or a prediction, computed using the criterion $\Xi_{h}^{n-1}$. Fig. 11 shows the adapted meshes obtained using the previous criterion. We plot in Fig. 12 the convergence of the error between the computed solution $\varphi_{h}$ and the exact solution $\varphi$ with respect to the equivalent space discretization $h_{e q}$. The error is plotted in the logarithmic scale and results show that the convergence slope is improved by mesh adaptation.

\subsection{Numerical results in the two-dimensional case}

In this section, we provide some numerical results of the equilibrium shapes of RBCs in the two-dimensional case. Since we do not dispose of an analytical expression describing the RBC's shape in the equilibrium state, a validation of the adaptive finite element method can be obtained by solving the reduced order problem. By varying the reduced area of the cell, we compare between the results obtained by the finite element solver and the results of the reduced order model as illustrated in the graph 6 . Concerning the numerical computations in the two-dimensional case, through a re-scaling step, we may provide shapes having a fixed perimeter $A_{0}$ and variable surface area. In all the results, we consider the RBC's perimeter at time $t=0$ to be $2 \pi$. We give a particular attention to the preservation of the area and the perimeter of the RBC, since it represents an important numerical difficulty related to the Eulerian methods.

We first consider the numerical simulation of a biconcave RBC having a reduced area $\gamma=0.75$. Regarding 


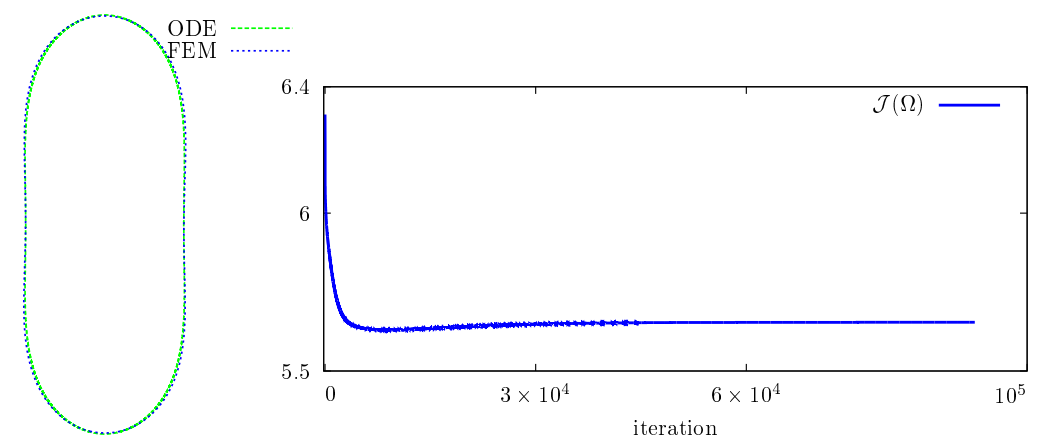

Figure 13: Evolution to the equilibrium shape of a RBC having $\gamma=0.75$ : (left) comparison between the finite element solution (FEM) and the solution given by the reduced model (ODE). (right) Minimization of the Canham-Helfrich energy.
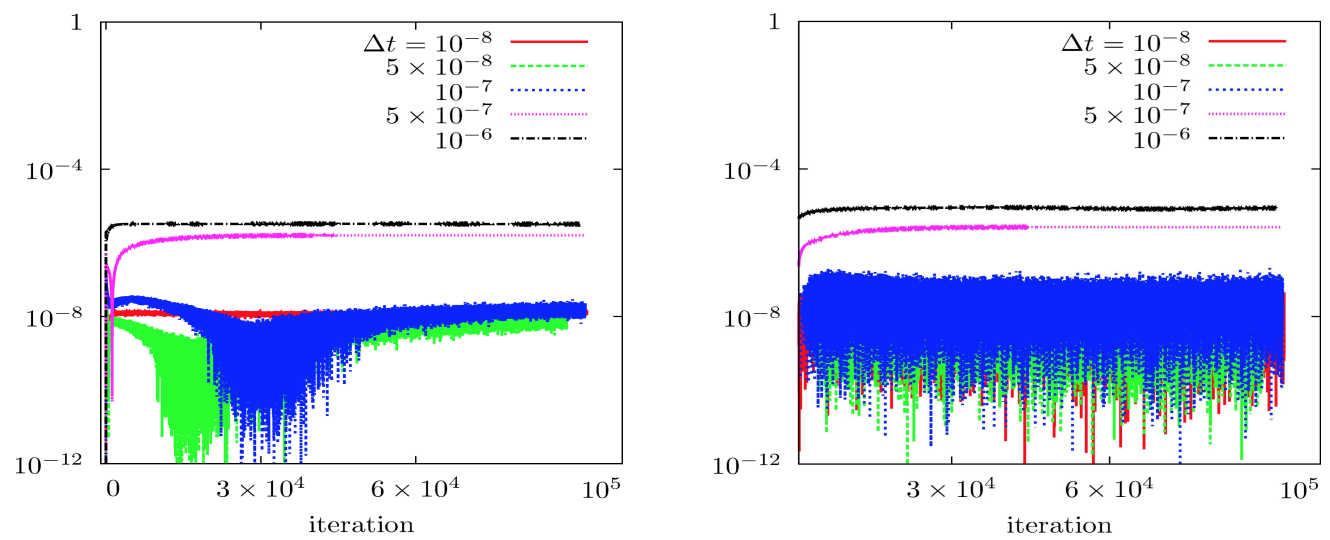

Figure 14: Evolution of the relative errors for different values of $\Delta t$. The y-coordinate is scaled logarithmically. (left) Relative error of the area. (right) Relative error of the perimeter.

the regularization procedure 2.3, we choose the regularization parameter $\varepsilon_{\mid K}=2.5 \times h_{\mid K}$ where $h_{\mid K}$ represents an estimation of the mesh size in the element $K \in \mathcal{T}_{h}$. Concerning the convergence to the steady state describing the RBC's equilibrium, Fig. 15 (right) depicts the convergence history showing the discrete $H^{-1}$ norm of the residual $\operatorname{res}_{h}^{n}$ as described in the section 3.3 Results show that the residual reaches a plateau that should decrease with respect to $\Delta t$. In a similar way, we plot in Fig. 16 the convergence history of the relative errors in the $L^{2}$ norm and the residual evaluated using the $L^{1}$ and $L^{2}$ norms for various values of the time step. An element that deserves more attention is the preservation of the perimeter and the area enclosed inside the cell. For a particular numerical computation, the quality of the numerical result and the preservation of the constraints are affected by the choice of time step $\Delta t$. Our concern here is to test the preservation of the constraints of perimeter and area for different time step sizes. In this numerical experiment, we focus on the equilibrium of the same RBC having a reduced area $\gamma=0.75$ starting from the same initial shape and using different time step sizes, see Fig. 13 (left) for an illustration of the equilibrium configuration of the cell. We plot in Fig. 14(left) and Fig. 14(right) the evolution of the relative error in the area and the perimeter. Numerical results show that errors are preserved remarkably well and they are smaller than $10^{-5 \%}$ when the time step size is smaller than $10^{-7}$. We verify also that the convergence is obtained for the two Lagrange multipliers $p$ and $\lambda$. Fig. 15 (left) shows their plots for a RBC having $\gamma=0.75$ and using a time step size $\Delta t=5 \times 10^{-9}$. The different simulations are all stopped when the residual $\left|\mathrm{res}_{h}^{n}\right|_{\mathrm{H}_{\mathrm{h}}^{-1}}$ reaches the tolerance criterion $\epsilon=10^{-5}$. The numerical results confirm, as expected, that the Lagrange multipliers converge to the constant values $p=3.3$ and $\lambda=-0.95$ in the steady state. In the following, the time step is set equal to $\Delta t=5 \times 10^{-7}$. We perform a qualitative comparison with the results obtained by solving the reduced order model 4.11. Regarding the numerical resolution of 4.11, we assume that the grid is uniform and we consider the spatial discretization parameter $N=10^{4}$ that corresponds to a mesh grid size $\Delta x=\frac{x_{f}}{N}$. The solution of the ordinary differential equation 4.10 having the same reduced area $\gamma=0.75$ as the RBC is obtained by setting the parameters $\lambda=-0.95, p=3.3$ and $w^{\prime}(0)=0.296$. A satisfactory agreement is observed with respect to the finite element 
solution in Fig. 13 (left). Fig. 13(right) plots the evolution of the Canham-Helfrich energy with respect to the iterations. The curve shows that the energy is decreasing before getting a stationary constant value equal to 5.68 .
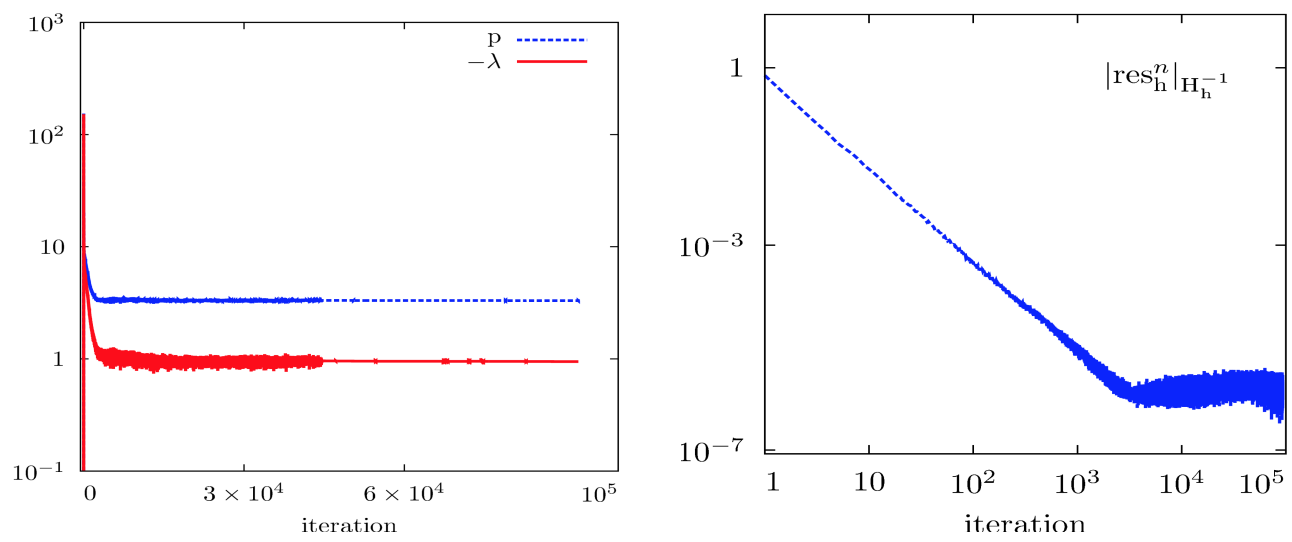

Figure 15: (left) Evolution of the Lagrange multipliers. The y-coordinate is scaled logarithmically. (right) Convergence of the residual res $_{\mathrm{h}}^{n}$ evaluated with the discrete $\mathrm{H}_{\mathrm{h}}^{-1}$ norm as a function of the iteration number. The coordinates are scaled logarithmically.
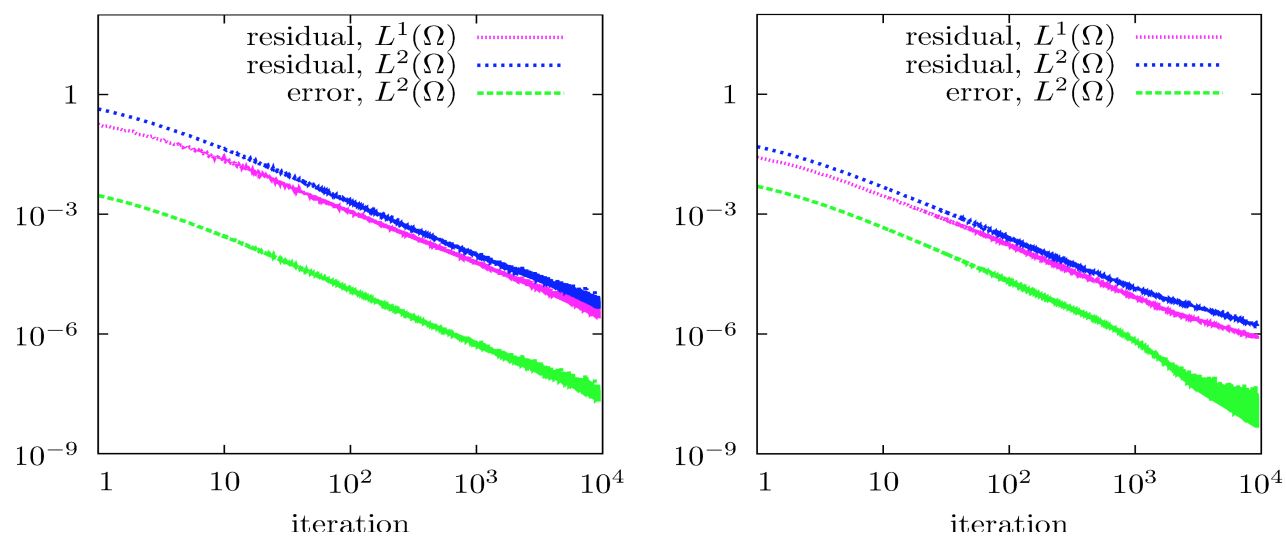

Figure 16: Convergence in several norms of the residual and the error using two different time step sizes: (left) $\Delta t=10^{-8}$ and (right) $\Delta t=10^{-7}$. Results are plotted in the logarithmic scale.

We now presents some other computational results that illustrate various shapes of RBCs and the corresponding reduced areas and energies of Canham and Helfrich. This experiment given in Fig 17 is designed to validate the equilibrium shape of a cell having a reduced area $\gamma=0.85$. The finite element results shows that the energy of Canham and Helfrich is decreasing with respect to the iterations, and the corresponding final energy value is 4.64 , as depicted in Fig 17 (right). The solution of the reduced model having the same reduced area $\gamma=0.85$ is obtained by using the following parameters: $N=10^{4}, \lambda=-0.95, p=3.3$ and $w^{\prime}(0)=0.48$. The two equilibrium shapes are almost the same, as shown in Fig 17 (left).

The next experiment concerns the equilibrium shape of a cell having a reduced area $\gamma=0.65$. The solution of the reduced model having the same reduced area is obtained by solving the ordinary differential equation 4.11 using the parameters $N=10^{4}, \lambda=-0.9, p=3.2$ and $w^{\prime}(0)=0.296$. We solve the finite element problem, and results illustrated in Fig[18(left) show that both forms are almost the same. The corresponding energy of Canham and Helfrich decreases to a constant value, and the final energy value is around 6.89 .

We consider in the next numerical test a cell with the reduced area $\gamma=0.6$. For the same reduced area, the solution obtained by solving the reduced order problem is obtained using the parameters $N=10^{4}, \lambda=-0.9, p=3.2$ and $w^{\prime}(0)=0.4$. A satisfactory agreement is observed with respect to the finite element solution in Fig. 19 (left). Fig. 19 (right) plots the minimization of the energy of Canham and Helfrich with respect to the iterations. The energy is decreasing before getting a stationary constant value around 7.4.

The next numerical test considers a RBC having a reduced area $\gamma=0.55$. The solution having the same reduced area obtained by solving the ordinary differential equation 4.11 is obtained by setting $N=10^{4}, \lambda=-0.95, p=3.3$ 


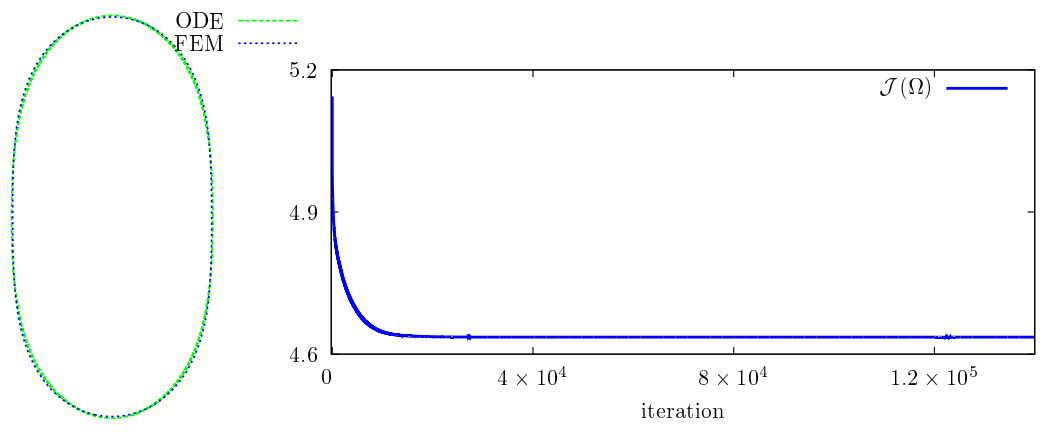

Figure 17: Evolution to the equilibrium shape of a RBC having $\gamma=0.85$ : (left) comparison between the finite element solution (FEM) and the solution of the reduced model (ODE). (right) Minimization of the Canham-Helfrich energy.

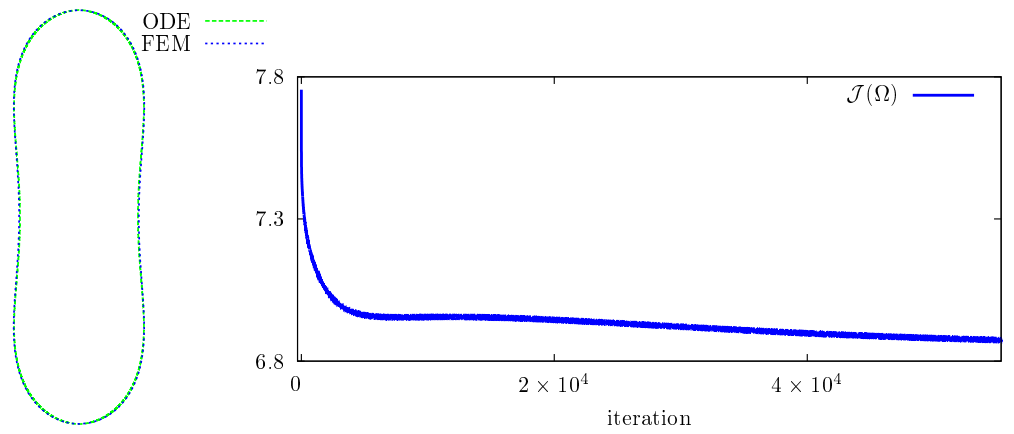

Figure 18: Evolution to the equilibrium shape of a RBC having $\gamma=0.85$ : (left) comparison between the finite element solution (FEM) and the solution of the reduced model (ODE). (right) Minimization of the Canham-Helfrich energy.

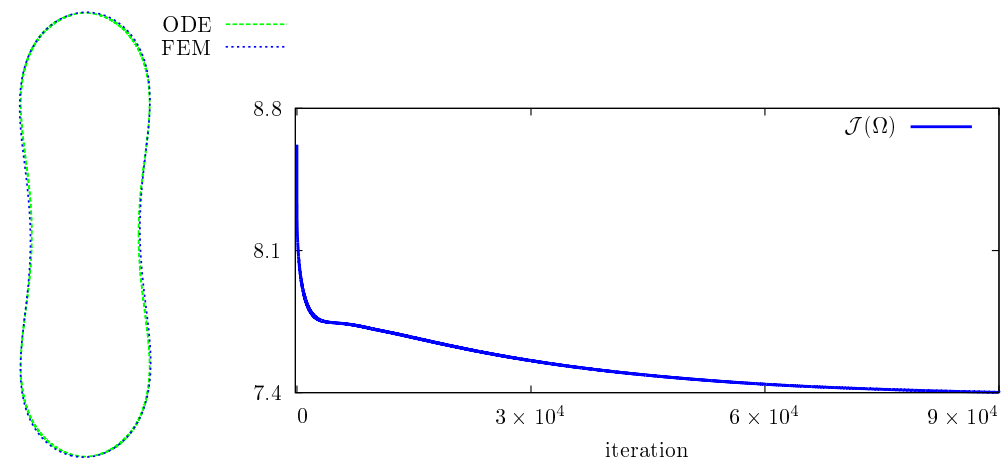

Figure 19: Evolution to the equilibrium shape of a RBC having $\gamma=0.60$ : (left) comparison between the finite element solution (FEM) and the solution of the reduced model (ODE). (right) Minimization of the Canham-Helfrich energy.

and $w^{\prime}(0)=0.48$. By comparing with the results of the finite element solver, we clearly notice the similarities between the two forms, as shown in Fig 20(left). The evolution of the energy is plotted in Fig. 20(right). Observe that the constant final value is equal to 8.04 .

In the final simulation, we provide a numerical validation of the equilibrium shape of a RBC having a reduced area $\gamma=0.49$. We obtain the solution of the reduced order model having the same shape parameter $\gamma$ by using the following numerical parameters $N=10^{4}, \lambda=0, p=3.3$ and $w^{\prime}(0)=0.55$. In Fig. 21(left), we overlay this solution with the shape obtained by the finite element computations. The similarity between the two shapes is observed. We show in Fig. 211(right) that the energy of Canham and Helfrich converges to a constant value equal to 9.2.

To summarize, the above numerical simulations in the two-dimensional case illustrate the robustness of the finite element solver modeling the static equilibrium of RBCs with the model introduced by Canham and Helfrich. The validation step is performed by comparing the finite element computations with the results of the reduced order 


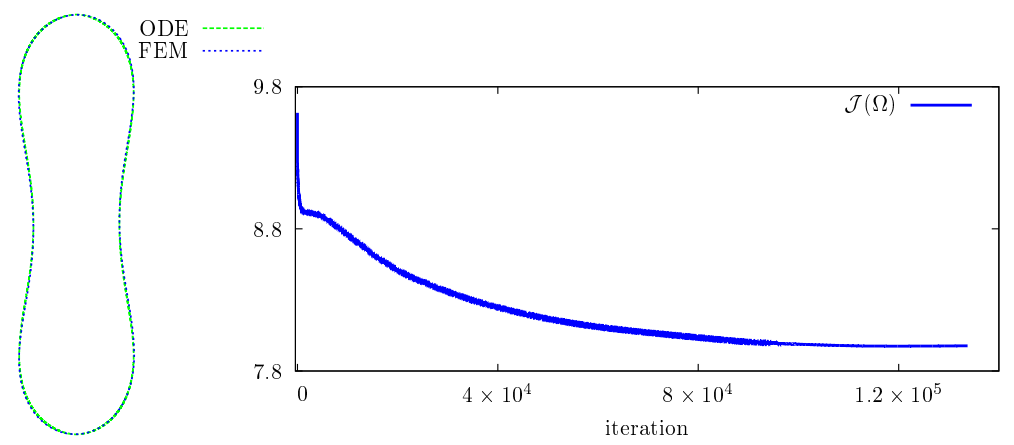

Figure 20: Evolution to the equilibrium shape of a RBC having $\gamma=0.55$ : (left) comparison between the finite element solution (FEM) and the solution of the reduced model (ODE). (right) Minimization of the Canham-Helfrich energy.

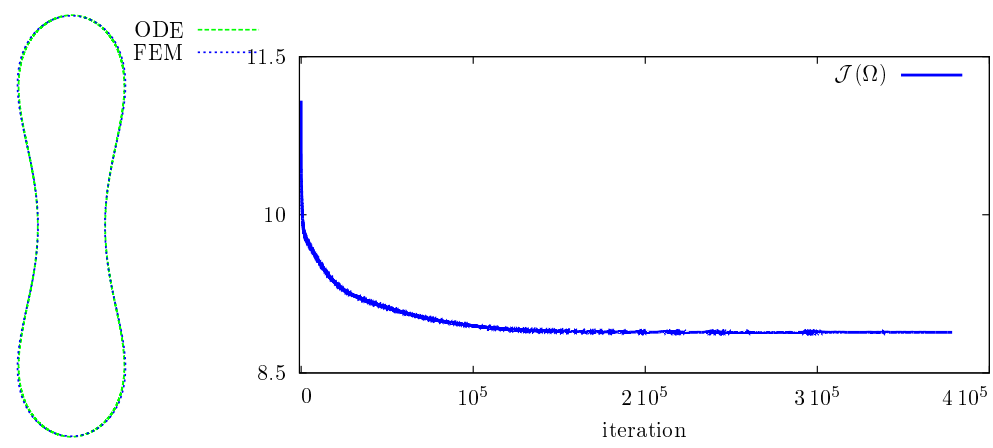

Figure 21: Evolution to the equilibrium shape of a RBC having $\gamma=0.49$ : (left) comparison between the finite element solution (FEM) and the solution of the reduced model (ODE). (right) Minimization of the Canham-Helfrich energy.

model. Furthermore, we superpose in Fig 22 some equilibrium shapes of RBCs having different reduced areas $\gamma$. These shapes are given by the finite element computations.

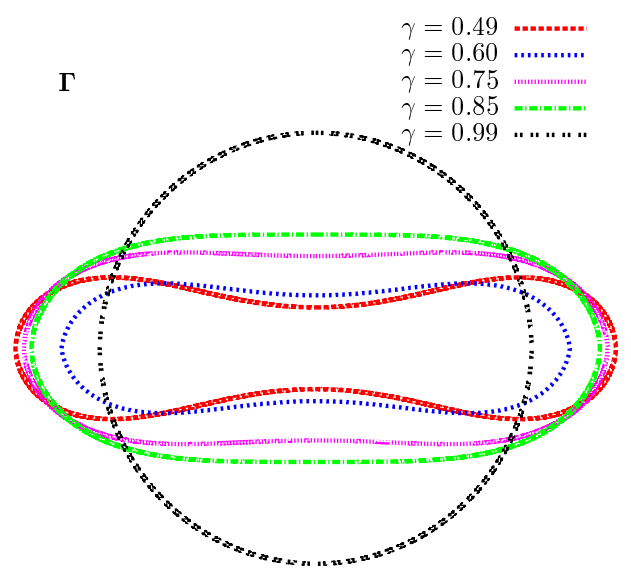

Figure 22: Two-dimensional case: overlay of some equilibrium shapes $\Gamma$ of RBCs with respect to their reduced areas $\gamma$. A dimensionless representation with a constant perimeter $A_{0}=2 \pi$ is provided.

Convergence properties of the numerical method. In this paragraph, we investigate numerically the convergence properties of prescribed finite element method. The spatial accuracy is studied by computing the error in the $L^{2}$ norm on successively refined meshes with respect to the reference solution obtained by solving the reduced order problem. The comparison is performed for three different RBCs having the reduced area $\gamma \in\{0.80,0.85$ and 0.95$\}$, respectively. Table 23. (left) depicts the computed error $\left\|\mathscr{H}_{\varepsilon}\left(\varphi_{\mathrm{ODE}}\right)-\mathscr{H}_{\varepsilon}\left(\varphi_{\mathrm{h}}\right)\right\|_{0,2, \Lambda}$ for successively refined meshes 
with respect to spatial resolution given by the equivalent mesh size $h_{\text {eq }}$, previously defined in section 5.2 .1 The finite element solution is called $\varphi_{\mathrm{h}}$, whereas $\varphi_{\mathrm{ODE}}$ represents the solution of the reduced order problem. The error history is displayed in Figure 23(right); it depicts the convergence properties and , in particular, the convergence rate of the numerical method. We notice that the error evolution disposes similar convergence rates for the different RBCs. By observing the slope in logarithmic scale, we can suggest that the error convergence follows $\mathcal{O}\left(h^{1+\alpha}\right)$ where $\alpha \in(0,1)$ represents a real parameter.

\begin{tabular}{|l||ccc|}
\hline \multicolumn{4}{|c|}{$\left\|\mathscr{H}_{\varepsilon}\left(\varphi_{\mathrm{ODE}}\right)-\mathscr{H}_{\varepsilon}\left(\varphi_{\mathrm{h}}\right)\right\|_{0,2, \Lambda}$} \\
\hline$h_{e q}$ & $\gamma=0.75$ & $\gamma=0.85$ & $\gamma=0.95$ \\
\hline \hline 0.052 & 0.03 & 0.027 & 0.015 \\
0.036 & 0.0085 & 0.0077 & 0.005 \\
0.017 & 0.0020 & 0.0018 & 0.00086 \\
0.011 & 0.00102 & 0.0008 & 0.00044 \\
0.006 & 0.0004 & 0.00022 & 0.000102 \\
\hline
\end{tabular}

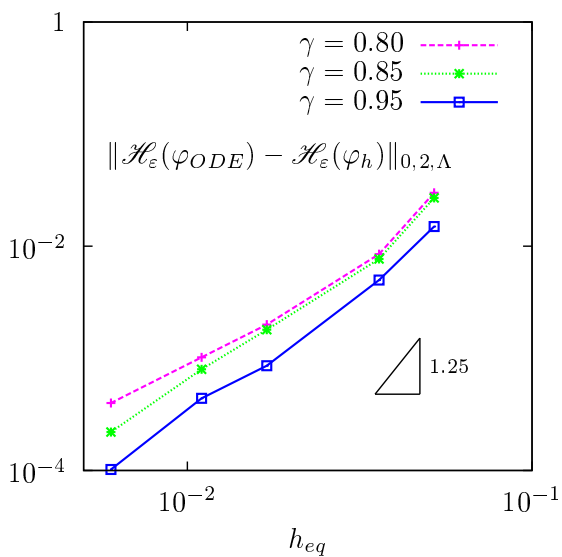

Figure 23: Convergence properties of the numerical method: evolution of the error, computed in the $L^{2}(\Lambda)$ norm, with respect to the equivalent mesh size $h_{e q}$ for several values of the reduced area $\gamma$.

\subsection{Numerical results in the three-dimensional axisymmetric case}
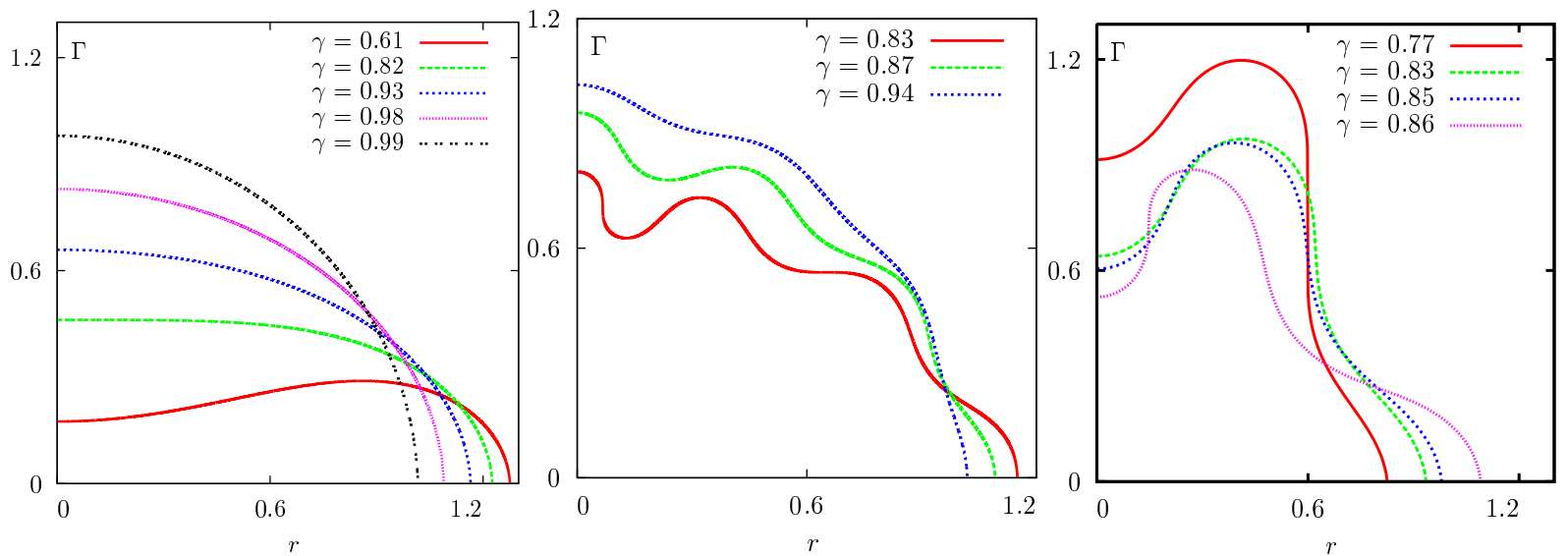

Figure 24: $3 \mathrm{D}$ axisymmetric case. (left) RBCs shapes having $i^{\star} \in\{0,1\}$. The corresponding values of the energy of Canham and Hefrich are respectively $\mathcal{J}(\Omega) \in\{55.48,40.08,31.24,28.08,24.65\}$, from inside to outside. (middle) RBCs shapes having $i^{*}=6$. They corresponds respectively to $\mathcal{J}(\Omega) \in\{79.02,83.23,84.5,83.34\}$, from inside to outside. (right) RBCs shapes having $i^{\star}=3$. They corresponds respectively to $\mathcal{J}(\Omega) \in\{79.77,80.47,80.59,96.52\}$, from inside to outside.

This section is concerned with the numerical investigation of RBCs shapes modeled in the three-dimensional case. We aim to give better insight into the properties of the Canham and Helfrich model in the three-dimensional case by further exploring our numerical tools, without the aim of providing a theoretical explanation of the physics underlying. We compute a three-dimensional axisymmetric solution where we assume both a rotational symmetry around the longitudinal axis and a symmetry with respect to the equatorial plane. Therefore, the computational domain covers the fourth of the RBC's shape, while the entire shape is reconstructed by both plannar and rotational symmetries. The modelization of the three-dimensional case with the axisymmetry is motivated by the great geometrical simplifications and the computational gain that one gets by doing two-dimensional computations to model three-dimensional shapes, allowing for an easier understanding of the underlying physics. We notice that the assembly of the global matrices in the three-dimensional axisymmetric case is automatically handled within 
the finite element environment Rheolef. The reduced order model is briefly described in Appendix A where we also provide the method used in the numerical approximation. A more detailed study was made by Wan et al. 5 . Numerical investigations show that we obtain the usual biconcave shapes of RBCs. Thanks to the simulations, we could bring to light new forms which are, up to our knowledge, unknown in the existing literature.

We focus on the computational domain that corresponds to one-quarter of the equatorial plane. We introduce an

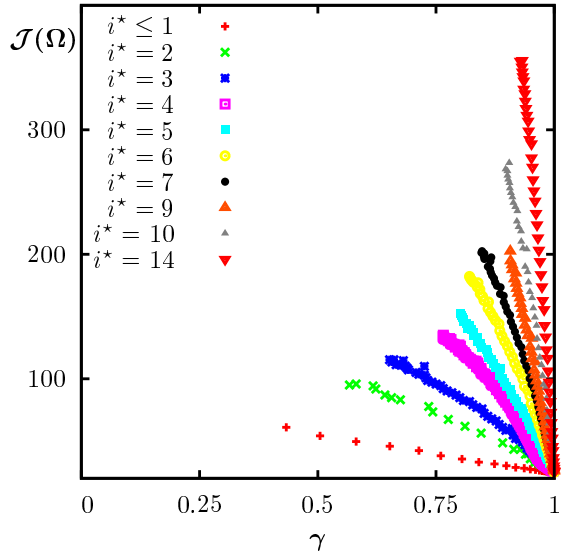

Figure 25: 3D axisymmetric case. Shape diagram of RBCs showing the energy of Canham and Helfrich $\mathcal{J}(\Omega)$ with respect to $\gamma$.

integer $i^{\star}$ that computes the number of point of inflection that corresponds to the points where concavity changes sign. For instance, a biconcave shape possess a value $i^{\star}=1$ and a sphere possesses a value $i^{\star}=0$. We plot in figure 24 some configurations of the equilibrium of RBCs corresponding to different values of $i^{\star} \in\{0,1,2,3\}$. All the shapes are dimensionless and they have a constant surface area equal to $4 \pi$. Let us consider, for example, the shapes having $i^{\star}=6$ : the reduced volume has values close to 1 when the shape is becoming closer to a spherical shape, see Fig 24 (middle). We perform a preliminary numerical study using several values of the reduced volume $\gamma$. A shape diagram is plotted in Fig 25] where each form of RBCs is represented by a point that the coordinates represent the reduced volume $\gamma$ and the corresponding energy $\mathcal{J}(\Omega)$, respectively. Numerical results show that a structure of branches is found, and each branch seems corresponding to fixed value of $i^{\star}$. Remark that several questions exist with respect to these energy levels, and we need a further study to understand and explain the diagram's structure and, in particular, how the membranes jump from an energy branch to another. Indeed, we proceed to change the reduced volume $\gamma$ of a RBC initially characterized by a shape parameter $i^{\star}$. A form change occurs when a critical value of gamma is reached, and accordingly it jumps from one branch to another. For a better visual effect, we perform a reconstruction of some three-dimensional shapes, and we give samples of the obtained forms in figure 26. The corresponding values of $i^{\star}, \gamma$ and $\mathcal{J}(\Omega)$ characterizing the shapes of RBCs are provided in the table below the shapes. Up to our knowledge, we don't know if some of these forms have been experimentally observed. However, we notice that other non-axisymmetric RBCs shapes obviously exist but they were not investigated in the present work. An investigation and a stability study of the presented shapes of RBCs will be investigated separately in a forthcoming paper.

\section{Conclusions}

In this paper, we present a mixed finite element method for the simulation of the equilibrium shapes of RBCs following the model proposed by Canham and Helfrich. These configurations are characterized by the minimization of the bending energy under the constraints of fixed volume and surface area. Our derivation is mostly based on a saddle point approach, with a level set method. Our framework allows the use of an anisotropic mesh adaptation technique that helps to better capture the RBC's membrane, and it allows more computational accuracy in the vicinity of the cell. Moreover, our method features the imposition of additional constraints via Lagrange multipliers technique combined with a posteriori mass corrections. Numerical results show the robustness of this strategy that enforces the mass preservation and improves the convergence properties of the method. We derive a reduced order model that describes the equilibrium shapes of RBCs in the two-dimensional case, and helps to further validate the finite element computations. We present a number of numerical examples in the two-dimensional case and the three-dimensional axisymmetric case that illustrate the main features of the numerical method. 


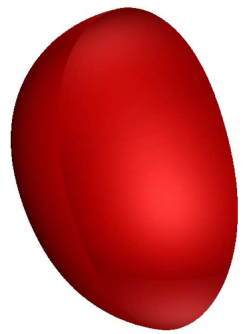

$\Gamma_{1}$
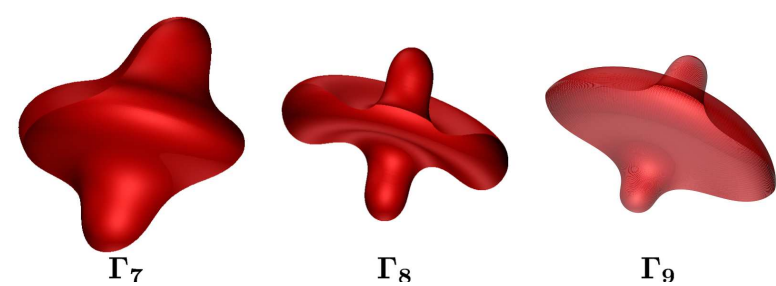

$\Gamma_{9}$

\begin{tabular}{|l||ccc|}
\hline \multicolumn{4}{|c|}{ 3D axisymmetric shapes } \\
\hline shape & $i^{\star}$ & $\gamma$ & $\mathcal{J}(\Omega)$ \\
\hline \hline$\Gamma_{1}$ & 0 & 0.91 & 30.05 \\
$\Gamma_{2}$ & 1 & 0.61 & 55.48 \\
$\Gamma_{3}$ & 3 & 0.86 & 79.77 \\
$\Gamma_{4}$ & 3 & 0.84 & 80.50 \\
$\Gamma_{5}$ & 3 & 0.77 & 96.52 \\
$\Gamma_{6}$ & 4 & 0.90 & 91.90 \\
\hline
\end{tabular}
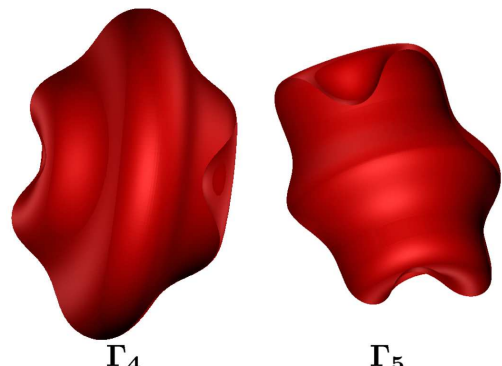

$\Gamma_{5}$
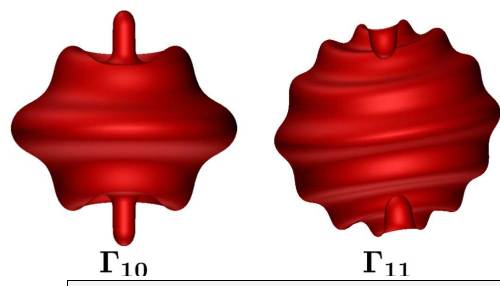

$\Gamma_{11}$

3D axisymmetric shapes

\begin{tabular}{|l||ccc|}
\hline shape & $i^{\star}$ & $\gamma$ & $\mathcal{J}(\Omega)$ \\
\hline \hline$\Gamma_{7}$ & 2 & 0.83 & 50.44 \\
$\Gamma_{8}$ & 2 & 0.61 & 100.61 \\
$\Gamma_{9}$ & 2 & 0.79 & 62.75 \\
$\Gamma_{10}$ & 4 & 0.78 & 147.6 \\
$\Gamma_{11}$ & 7 & 0.89 & 201.23 \\
$\Gamma_{12}$ & 11 & 0.94 & 240.77 \\
\hline
\end{tabular}
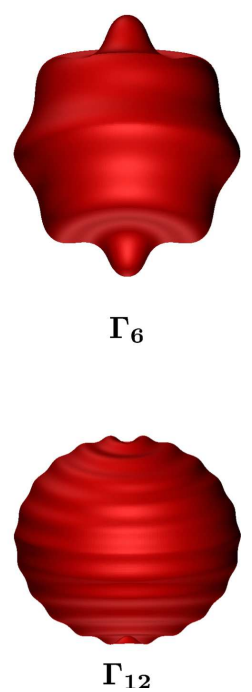

$\Gamma_{12}$

Figure 26: 3D axisymmetric case. Overlay of some shapes of RBCs obtained by the numerical simulations.

\section{Acknowledgments}

The authors gratefully acknowledge the partial support by the National Center for Scientific Research (CNRS) through a doctoral grant, by the French National Research Agency (ANR) through the financial grant, "MOSICOB project", by the Joseph Fourier University, and by the Swiss Federal Institute of Technology in Zürich.

\section{Appendix A. Reduced order model in the three-dimensional axisymmetric case}

A detailed description of the derivation of the reduced model in the three-dimensional axisymmetric case is available in [4, 5]. In what follows, only the numerical method is described. A parametric representation, i.e. $z=h(r)$ with $r \in\left(0, r_{f}\right)$, is used to describe the cell membrane, where $r$ is the radial distance and $z$ represents the coordinate perpendicular to the equatorial plane. A procedure similar to the two-dimensional Cartesian case can be used, where $w(r)=h^{\prime}(r)$. The quantity $\kappa(r)=\frac{w(r)}{r \sqrt{1+w^{2}(r)}}$ allows to describe curvature component comming from the three-dimensional modelization, and the mean curvature is given by $H(r)=-2 \kappa(r)-r \kappa^{\prime}(r)$. The curvature is singular on the longitudinal axis and we need to use a Taylor expansion around $r=0$. Hence, we introduce a small parameter $\varepsilon$ that we use equal to $10^{-7}$ in the numerical simulations, and the problem is solved in the interval $\left[\varepsilon, r_{f}\right]$. Therefore, the reduced order model is given in the three-dimensional axisymmetric case by:

$$
\begin{aligned}
& \kappa^{\prime \prime}=\frac{P(\kappa)-\kappa\left(r \kappa^{\prime}+\kappa\right)^{2}}{2\left(1-r^{2} \kappa^{2}\right)}-\frac{3 \kappa^{\prime}}{r}, \quad \text { for } r \in\left(\varepsilon, r_{f}\right) \\
& \kappa(\varepsilon)=w^{\prime}(0) \quad \text { and } \quad \kappa^{\prime}(\varepsilon)=0,
\end{aligned}
$$

where $P(\cdot)$ represents a polynomial function. Regarding the numerical resolution, we introduce $y(r)=\left(y_{1}, y_{2}\right)^{T}=$ $\left(\kappa(r), \kappa^{\prime}(r)\right)^{T}$ and $f(y, r)=\left(y_{2}, \frac{P\left(y_{1}\right)-y_{1}\left(r y_{2}+y_{1}\right)^{2}}{2\left(1-r^{2} y_{1}^{2}\right)}-\frac{3 y_{2}}{r}\right)^{T}$. Let us consider the initial data $y_{0}=\left(w_{0}^{\prime}, 0\right):=$ 
$\left(w^{\prime}(0), 0\right)$ and the boundary condition at $r=\varepsilon$ given by $y_{\varepsilon}=\left(w_{0}^{\prime},-\varepsilon w_{0}^{\prime 3}\right)$. The problem reads

$$
y^{\prime}=f(y, r), \quad \text { for } r \in\left(\varepsilon, r_{f}\right) \quad \text { and } \quad y(\varepsilon)=y_{\varepsilon} .
$$

We use a similar approach to that of the two-dimensional Cartesian case, and we have

$$
w(r)=\frac{r \kappa}{\sqrt{1-r^{2} \kappa^{2}}}, \quad \text { for } r \in\left(\varepsilon, r_{f}\right) \quad \text { and } \quad h(r)=\int_{r_{f}}^{r} w(r) \mathrm{d} r .
$$

[1] G. Allaire and F. Jouve. Minimum stress optimal design with the Level Set method. International Journal of Boundary Elements and Applications (accepted), 2007.

[2] S.M. Allen and J.W. Cahn. A microscopic theory for antiphase moundary motion and its application to antiphase domain coarsening. Acta Metal., 27:1085-1095, 1979.

[3] J. Arrow, L. Hurwicz, and H. Uzawa. Studies in linear and nonlinear programming. Stanford University Press, pages 127-132, 1958.

[4] T. K.-K. Au and T. Y.-H. Wan. Analysis on an ODE arisen from studying the shape of a red blood cell. Journal of Mathematical Analysis and Applications, 282(1):279-295, 2003

[5] T. K.-K. Au and T. Y.-H. Wan. Pysical parameters for biconcave shape vesicles. Physical Review Letters, February 2, 2008.

[6] T. Banham, B. Li, and Y. Zhao. Pattern formation by phase-field relaxation of bending energy with fixed surface area and volume. Phys. Rev. E, 90:033308, Sep 2014.

[7] T. Biben, K. Kassner, and C. Misbah. Phase-Field approach to three-dimensional vesicle dynamics. Phys. Rev. E, $72,2005$.

[8] A. Bonito, R.H. Nochetto, and M.S. Pauletti. Dynamics of biomembranes: Effect of the bulk fluid. Mathematical Modelling of Natural Phenomena, 6:25-43, 12011.

[9] H. Borouchaki, P.L. George, F. Hecht, P. Laug, and E. Saltel. Delaunay mesh generation governed by metric specifications. part i. algorithms. Finite Elements in Analysis and Design, 25(1-2):61-83, 1997.

[10] H. Borouchaki, P.L. George, and B. Mohammadi. Delaunay mesh generation governed by metric specifications part ii. applications. Finite Elements in Analysis and Design, 25:85-109, 1997.

11] Robert L. Bryant. A duality theorem for willmore surfaces. Journal of Differential Geometry, 20(1):23-53, 1984.

[12] P.B. Canham. The minimum energy of bending as a possible explanation of the biconcave shape of the human red blood cell. $J$. Theor. Biol., 26:61-81, 1970.

[13] G.-H. Cottet, E. Maitre, and T. Milcent. Eulerian formulation and level-set models for incompressible fluid-structure interaction. Math. Model. Numer. Anal., 42:471-492, 2008.

[14] V. Cristini, J. Bawzdziewicz, and M. Loewenberg. Drop breakup in three-dimensional viscous flows. Physics Fluids, 10:1781-1783, 1998.

[15] H.J. Deuling and W.Helfrich. The curvature elasticity of fluid membranes: a catalog of vesicle shapes. J. Phys. (Paris), 37:1335$1345,1976$.

[16] H.J. Deuling and W.Helfrich. Red blood cell shapes as explained on the basis of curvature elasticity. Biophys. J., 16:861-868, 1976.

[17] H.J. Deuling and W.Helfrich. A theoretical explanation for the myelin shapes of red blood cells. Blood Cells, 3:713-720, 1977.

[18] M. P. do Carmo. Differential geometry of curves and surfaces. Prentice-Hall Englewood Cliffs, NJ, 1976.

[19] Q. Du, M. Li, and C. Liu. Analysis of a Phase Field Navier-Stokes vesicle-fluid interaction model. Discrete and continuous dynamical system - series $B, 8: 3,2007$.

[20] Q. Du, C. Liu, R. Ryham, and X. Wang. Energetic variational approaches in modeling vesicle and fluid interactions. Physica D: Nonlinear Phenomena, 238(9-10):923-930, 2009.

[21] Q. Du, C. Liu, and X. Wang. A phase field approach in the numerical study of the elastic bending energy for vesicle membranes. J. Comput. Phys., 198:450-468, 2004.

[22] Q. Du, C. Liu, and X. Wang. Simulating the deformation of vesicle membranes under elastic bending energy in three dimension. J. of Comput. Phys., 212:757-777, 2006.

[23] G. Dziuk. Computational parametric willmore flow. Numerische Mathematik, 111(1):55-80, 2008.

[24] J.W. Eaton, D. Bateman, and S. Hauberg. GNU Octave version 3.0.1 manual: a high-level interactive language for numerical computations. Create Space Independent Publishing Platform, 2009. ISBN 1441413006.

[25] J. Elschner. C. pozrikidis, boundary integral and singularity methods for linearized viscous flow. cambridge university press 1992. ZAMM - Journal of Applied Mathematics and Mechanics, 74(2):104-104, 1994.

[26] D. Enright, R. Fedkin, J. Ferziger, and I. Mitchell. A hybrid partical Level Set method for improved interface capturing. J. Comput. Phys., 183:83-116, 2002.

[27] Y.C. Evans and J. Spruck. Motion of Level Sets by mean curvature. J. Differential Geo, 33(8):635-681, 1991.

[28] L. Formaggia and S. Perotto. New anisotropic a priori error estimates. Numer. Math., 89:641-667, 2001.

[29] M. Fortin and R. Glowinski. , méthodes de lagrangien augmenté. applications à la résolution numrique de problmes aux limites. Mthodes Mathématiques de l'Informatique 9, page 170, 1982.

[30] P.L. George and H. Borouchaki. Triangulation de Delaunay et maillage: applications aux éléments finis. Hermes, 1997.

[31] C. Geuzaine and J.-F. Remacle. Gmsh: a three-dimensional finite element mesh generator with built-in pre- and post-processing facilities. Int. J. Numer. Methods Engrg., 79(11):1309-1331, 2009.

[32] R. Glowinski. Numerical methods for nonlinear variational problems. Spinger-Verlag, New York, 1984.

[33] R. Glowinski and P. Le Tallec. Augmented lagrangians and operator-splitting methods in nonlinear mecanics. SIAM, Philadelphia, 1989.

[34] G. Golub and W. Kahan. Calculating the singular values and pseudo-inverse of a matrix. Journal of the Society for Industrial and Applied Mathematics Series B Numerical Analysis, 2(2):205-224, 1965.

[35] G.H. Golub. Matrix computations. Johns Hopkins University Press, 3rd edition, 101996.

[36] G.H. Golub and C. Reinsch. Singular value decomposition and least squares solutions. Numerische Mathematik, 14(5):403-420, 1970.

[37] D. Gueyffier, J. Li, A. Nadim, R. Scardovelli, and S. Zaleski. Volume-of-fluid interface tracking with smoothed surface stress methods for three-dimensional flows. J. Comput. Phys., 152:423-456, 1999. 
[38] F. Hecht. BAMG: bidimensional anisotropic mesh generator, 2006. http://www.rocq.inria.fr/gamma/cdrom/www/bamg/eng.htm.

[39] Z.-N. Hu. New approach on the general shape equation of axisymetric vesicles. Mod. Phys. Lett. B, 1999.

[40] W. Huang. Metric tensors for anisotropic mesh generation. J. Comput. Phys., 204:633-665, 2005.

[41] W. Huang and X. Li. An anisotropic mesh adaptation method for the finite element solution of variational problems. Finite Elements in Analysis and Design, 46:61-73, 2010.

[42] S. Ianniello and A. Di Mascio. A self-adaptive oriented particles level-set method for tracking interfaces. Journal of Computational Physics, 229(4):1353 - 1380, 2010.

[43] J.Hu and Z. Ou-Yang. Shape equations for the axisymetric vesicles. Phys.Rev.E, 47:461-467, 1993.

[44] F. Julicher and U. Seifert. Shape equations for axisymmetric vesicles: A clarification. Phys.Rev.E, 49(5):4728-4731, 1994.

[45] B. Kaoui, G. H. Ristow, I. Cantat, C. Misbah, and W. Zimmermann. Lateral migration of a two-dimensional vesicle in unbounded poiseuille flow. Phys. Rev. E, 77:021903, Feb 2008.

[46] R.E. Khayat. Three-dimensional boundary-element analysis of drop deformation for newtonian and viscoelastic systems. Int. J. Numer. Meth. Fluids, 34:241-275, 2000.

[47] A. Laadhari, C. Misbah, and P. Saramito. On the equilibrium equation for a generalized biological membrane energy by using a shape optimization approach. Physica D, 239:1567-1572, 2010.

[48] A. Laadhari, P. Saramito, and C. Misbah. Improving the mass conservation of the Level Set method in a Finite Element context. Compt. Rend. Math. Ser. I, 348:535-540, 2010.

[49] A. Laadhari, P. Saramito, and C. Misbah. Vesicle tumbling inhibited by inertia. Physics of Fluids, 24:031901, 2012.

[50] R.J. Leveque. High-resolution conservative algorithms for advection in incompressible flow. SIAM J. Numer. Anal., 33:627-665, 1996.

[51] T. Menard, S. Tanguy, and A. Berlemont. Coupling level set/vof/ghost fluid methods: Validation and application to 3d simulation of the primary break-up of a liquid jet. International Journal of Multiphase Flow, 33(5):510 - 524, 2007.

[52] L. Miao, U. Seifert, M. Wortis, and H. Dobereiner. Budding transitions of fluidbilayer vesicles - the effect of area-difference elasticity. Phys. Rev. E, 49:5389-5407, 1994.

[53] F. Murat and J. Simon. Quelques résultats sur le contrôle par un domaine géométrique. Technical Report LA 189, Lab. d'Analyse Numérique, Univ. Paris VI, 1974.

[54] J.-C. Nave, R.R. Rosales, and B. Seibold. A gradient-augmented level set method with an optimally local, coherent advection scheme. Journal of Computational Physics, 229(10):3802 - 3827, 2010.

[55] S. Osher and R. Fedkiw. Level Set methods: An overview and some recent results. J., 2005.

[56] S. Osher and J.A. Sethian. Front propaging with curvature deppend speed: Agorithms based on Hamilton-Jacobi formulations. J. Comput. Phys., 79(12), 1988.

[57] Z.-C. Ou-Yang and W. Helfrich. Bending energy of vesicle membranes: general expressions for the first, second and third variation of the shape energy and applications to spheres and cylinders. Phys. Rev. A, 39(10):5280-5288, 1989.

[58] M. Owkes and O. Desjardins. A computational framework for conservative, three-dimensional, unsplit, geometric transport with application to the volume-of-fluid (vof) method. Journal of Computational Physics, 270:587-612, 2014.

[59] C.S. Peskin. The immersed boundary method. Acta Numerica, 11:1-39, 2002.

[60] F.B. Sachse. Computational Cardiology: Modeling of Anatomy, Electrophysiology, and Mechanics. Springer-Verlag, Berlin, 2004.

[61] S. A. Safran. Statistical Thermodynamics of Surfaces, Interfaces and Membranes. Frontier in Physics Vol 90. Addison-Wesley Publishing Company, Reading, MA, 1994.

[62] D. Salac and M. Miksis. A level set projection model of lipid vesicles in general flows. J. Comput. Phys., 230:8192-8215, 2011.

[63] H. Samuel. A level set two-way wave equation approach for eulerian interface tracking. Journal of Computational Physics, 259(0):617 - 635, 2014.

[64] P. Saramito. Efficient C++ Finite Element computing with Rheolef. $\quad$ CNRS-CCSD ed., 2014. http://cel.archives-ouvertes.fr/cel-00573970.

[65] U. Seifert. Configurations of fluid membranes and vesicles. Adv. in Phys., 46:13-137, 1997.

[66] U. Seifert, K. Berndl, and R. Lipowsky. Shape transformations of vesicles: Phase diagram for spontaneous- curvature and bilayercoupling models. Phys. Rev. A, 44:1182-1202, Jul 1991.

[67] J. Simon. Differentiation with respect to the domain in boundary value problems. J. Num. Funct. Anal. Optim., 2(7-8):649-687, 1980.

[68] S.K. Veerapaneni, D. Gueyffier, D. Zorin, and G. Biros. A boundary integral method for simulating the dynamics of inextensible vesicles suspended in a viscous fluid in 2d. Journal of Computational Physics, 228(7):2334 - 2353, 2009.

[69] S.K. Veerapaneni, R. Raj, G. Biros, and P.K. Purohit. Analytical and numerical solutions for shapes of quiescent 2d. vesicles. International Journal of Non-Linear Mechanics, 44(3):257-262, 2009.

[70] P. M. Vlahovska, T. Podgorski, and C. Misbah. Vesicles and red blood cells in flow: From individual dynamics to rheology. Compte Rendus Physique, 10:775-789, 2009.

[71] N. Walani, J. Torres, and A. Agrawal. Anisotropic spontaneous curvatures in lipid membranes. Phys. Rev. E, 89:062715, Jun 2014.

[72] W.Helfrich. Elastic properties of lipid bilayers: theory and possible experiments. Z. Naturforsch. Teil C, 28:693-703, 1973.

[73] T.J. Willmore. Riemannian Geometry. Clarendon, Oxford, 1993.

[74] G.H. Yeoh and J. Tu. Computational Techniques for Multiphase Flows. 2010.

[75] S. Zalesak. Fully multidimensional flux-corrected trasport algorithms for fluids. J. Comput. Phys., 31:335-362, 1979.

[76] D. Zhang, C. Jiang, D. Liang, Z. Chen, Y. Yang, and Y Shi. A refined volume-of-fluid algorithm for capturing sharp fluid interfaces on arbitrary meshes. Journal of Computational Physics, 274(0):709 - 736, 2014.

[77] L. Zhao, X. Bai, T. Li, and J. J. R. Williams. Improved conservative level set method. International Journal for Numerical Methods in Fluids, 75(8):575-590, 2014.

[78] L. Zhao, J. Mao, X. Bai, X. Liu, T. Li, and J.J.R. Williams. Finite element implementation of an improved conservative level set method for two-phase flow. Computers and Fluids, 100(0):138 - 154, 2014.

[79] W.-M. Zheng and J. Liu. Helfrich shape equation for axisymmetric vesicles as a first integral. Phys. Rev. E, 48(4):2856-2860, 1993.

[80] O.-Y. Zhong-Can and W. Helfrich. Bending energy of vesicle membranes: general expressions for the first, second and third variation of the shape energy and applications to spheres and cylinders. Phys. Rev. A, 39(10):5280-5288, 1989. 